ARTICLE

DOI: $10.1038 / s 41467-018-06833-7$

\title{
Myh10 deficiency leads to defective extracellular matrix remodeling and pulmonary disease
}

Hyun-Taek Kim', Wenguang Yin (1) 1, Young-June Jin², Paolo Panza (1) 1, Felix Gunawan (1) 1, Beate Grohmann', Carmen Buettner ${ }^{1}$, Anna M. Sokol (10 ${ }^{3}$, Jens Preussner ${ }^{4}$, Stefan Guenther ${ }^{4}$, Sawa Kostin (1D ${ }^{5}$, Clemens Ruppert ${ }^{6}$, Aditya M. Bhagwat ${ }^{7}$, Xuefei Ma ${ }^{8}$, Johannes Graumann (10 ${ }^{3,9}$, Mario Looso ${ }^{4}$, Andreas Guenther ${ }^{6}$, Robert S. Adelstein ${ }^{8}$, Stefan Offermanns ${ }^{2,9}$ \& Didier Y.R. Stainier ${ }^{1,9}$

Impaired alveolar formation and maintenance are features of many pulmonary diseases that are associated with significant morbidity and mortality. In a forward genetic screen for modulators of mouse lung development, we identified the non-muscle myosin II heavy chain gene, Myh10. Myh10 mutant pups exhibit cyanosis and respiratory distress, and die shortly after birth from differentiation defects in alveolar epithelium and mesenchyme. From omics analyses and follow up studies, we find decreased Thrombospondin expression accompanied with increased matrix metalloproteinase activity in both mutant lungs and cultured mutant fibroblasts, as well as disrupted extracellular matrix (ECM) remodeling. Loss of Myh10 specifically in mesenchymal cells results in ECM deposition defects and alveolar simplification. Notably, MYH10 expression is downregulated in the lung of emphysema patients. Altogether, our findings reveal critical roles for Myh10 in alveologenesis at least in part via the regulation of ECM remodeling, which may contribute to the pathogenesis of emphysema.

\footnotetext{
${ }^{1}$ Department of Developmental Genetics, Max Planck Institute for Heart and Lung Research, Bad Nauheim 61231, Germany. ${ }^{2}$ Department of Pharmacology, Max Planck Institute for Heart and Lung Research, Bad Nauheim 61231, Germany. ${ }^{3}$ Scientific Service Group of Biomolecular Mass Spectrometry, Max Planck Institute for Heart and Lung Research, Bad Nauheim 61231, Germany. ${ }^{4}$ ECCPS Bioinformatics and Deep Sequencing Platform, Max Planck Institute for Heart and Lung Research, Bad Nauheim 61231, Germany. ${ }^{5}$ Scientific Service Group of Morphometry, Max Planck Institute for Heart and Lung Research, Bad Nauheim 61231, Germany. ${ }^{6}$ Biobank, University of Giessen \& Marburg Lung Center (UGLMC), Giessen 35392, Germany. ${ }^{7}$ Bioinformatics Core, Weill Cornell Medicine - Qatar, Doha PO 24144, Qatar. ${ }^{8}$ Laboratory of Molecular Cardiology, National Heart, Lung and Blood Institute, National Institutes of Health, Bethesda MD 20814, USA. ${ }^{9}$ German Centre for Cardiovascular Research (DZHK), Partner Site Rhine-Main, Frankfurt 60323, Germany. Correspondence and requests for materials should be addressed to H.-T.K. (email: hyun-taek.kim@mpi-bn.mpg.de) or to D.Y.R.S. (email: didier.stainier@mpi-bn.mpg.de)
} 
ung development is subdivided into five chronologically and structurally distinct stages: embryonic, pseudoglandular, canalicular, saccular, and alveolarization ${ }^{1,2}$. During the embryonic stage, the lung bud arises from the anterior foregut endoderm. From the pseudoglandular to canalicular stages, the lung undergoes branching morphogenesis to form a tree-like tubular structure. At the saccular stage, expansion and thinning of the alveolar walls lead to a marked decrease in interstitial tissue as a prerequisite for postnatal gas exchange. Finally, the lung greatly expands its alveolar surface by alveolarization, a process by which alveolar sacs are repeatedly partitioned through septation. Alveolar sac septation is a complex process, which requires interactions between lung epithelial and mesenchymal cells ${ }^{3-7}$. Although several studies have focused on these epithelial-mesenchymal interactions, the underlying mechanisms are still poorly understood.

Non-muscle myosin II (NM II) plays fundamental roles in the maintenance of cell morphology, cell adhesion, and migration, as well as cell division ${ }^{8-10}$. NM II molecules consist of three peptide pairs: a pair of NM II heavy chains (NMHC II), a pair of regulatory light chains (RLCs), and a pair of essential light chains (ELCs). In mammals, three different genes, Myosin heavy chain 9 (MYH9), MYH10, and MYH14, encode the NMHC II proteins, NMHC IIA, NMHC II-B, and NMHC II-C, respectively ${ }^{11,12}$. NM II proteins are highly expressed during lung development ${ }^{13}$, and their inhibition using blebbistatin leads to defects in branching morphogenesis and epithelial cell shape and orientation ${ }^{14}$. To date, however, genetic studies of NM II genes during lung development and homeostasis have not been conducted.

Many pulmonary diseases including chronic obstructive pulmonary disease (COPD) ${ }^{15,16}$ and interstitial lung disease (ILD) ${ }^{17}$ are associated with significant morbidity and mortality due to impaired alveolar formation and maintenance. Emphysema is one form of COPD that results from the enzymatic destruction of lung extracellular matrix (ECM) components, including elastin and collagen, thereby leading to destruction of alveolar walls and airspace enlargement ${ }^{15,16,18}$. Recently, several animal models have been established to study the pathophysiology of emphysema, including exposure to cigarette smoke and protease instillation, as well as genetic manipulation ${ }^{18-20}$. The pathophysiological role of NM II in pulmonary emphysema, however, has not been investigated.

Here, starting with a forward genetic screen in mouse, we reveal an unexpected role for the NM II-associated actomyosin network in ECM remodeling during lung development and disease. Notably, our results from animal models and human patients suggest that alterations of the actomyosin network by loss of MYH10 function contribute to the pathogenesis of emphysema and may provide a promising target for preventive care of emphysema.

\section{Results}

Identification of lung mutants following ENU mutagenesis. To identify novel factors regulating early postnatal lung development in mouse, we carried out a forward genetic screen using $N$-ethyl$N$-nitrosourea (ENU) mutagenesis ${ }^{21}$. From screening the F3 progeny of $170 \mathrm{G} 1$ mice, we identified eight families exhibiting a recessive alveolar collapse phenotype. Mutant newborns from one of these families were cyanotic and exhibited respiratory distress with full penetrance (Fig. 1a, Supplementary Movie 1). As their lungs did not inflate, mutant pups died within $24 \mathrm{~h}$ after birth (Figs. 1b, c). Histological and morphometric analyses revealed reduced alveolar spaces and thick alveolar septal walls (Fig. 1d), along with dilated cardiomyopathy and hydrocephalus ${ }^{22}$ (Supplementary Fig. 1a, b). We observed no histological differences between wild-type and mutant littermate lungs at the pseudoglandular stage. However, from embryonic day (E) 17.5 onward, the mutant lungs displayed reduced saccular spaces and more abundant mesenchyme compared with wild-type siblings (Fig. 1e).

To investigate whether the increased tissue density observed in the mutant lungs resulted from increased cell proliferation, altered apoptosis, or differentiation defects, we performed immunostaining using two proliferation markers, proliferating cell nuclear antigen (PCNA) and phospho-histone 3 ( $\mathrm{pH} 3)$, and quantified positive cells from E17 to postnatal day 0 (P0). To differentiate between mesenchymal and epithelial cells, we costained for the epithelial cell markers NKX2-1 and E-cadherin (Ecad). Interestingly, both epithelial and mesenchymal cells exhibited increased cell proliferation in mutant lungs compared with wild-type siblings (Supplementary Fig. 1c, d, 2a, b), explaining at least in part the observed increase in tissue density. A minor increase in the number of apoptotic cells was also observed in mutant lungs compared with wild-type siblings (Supplementary Fig. 1c, 2c), suggesting that loss of mutant cells does not contribute in a major way to the observed phenotypes.

Next, we examined the differentiation status of mutant epithelial and mesenchymal cells by staining for several marker proteins. Interestingly, although the number of Vimentin-positive fibroblasts $^{23}$ was dramatically decreased in mutant lungs, unaltered nerve/glial antigen (NG) 2-positive pericyte-like cells ${ }^{23}$ and endothelial cell numbers were observed in mutant lungs compared with wild-type siblings (Supplementary Fig. 1d). Moreover, mutant lungs exhibited an increase in the number of AT2 (alveolar epithelial type II) cells and AT2 cell marker (Sftpa, $S f t p c, A b c a 3$ ) expression levels (Supplementary Fig. 1e, 2d, e). Concurrently, we found a marked decrease in the expression levels of AT1 (alveolar epithelial type I) cell markers (homeodomin-only protein homeobox (HOPX), Podoplanin (PDPN), and receptor for advanced glycation end products (RAGE) $)^{24}$, as well as of a mature AT2 cell marker (lysosome-associated membrane glycoprotein (LAMP) 3$)^{25}$ (Supplementary Fig. 1e, f, 2e).

Immature AT2 cells store glycogen, which is converted into surfactant in lamellar bodies as these cells differentiate ${ }^{26}$. To examine the differentiation state of AT2 cells, we performed periodic acid-Schiff (PAS) staining, which revealed a substantial increase in the number of PAS-positive AT2 cells in P0 mutant lungs (Fig. 1f). By transmission electron microscopy (TEM), wildtype AT2 cells contained numerous lamellar bodies and apical microvilli at E18.5, whereas mutant AT2 cells displayed abundant glycogen, but fewer lamellar bodies and microvilli, indicating that they failed to differentiate (Fig. 1g). In addition, whereas AT1 cells in wild-type lungs covered the alveolar surface and exhibited a flattened morphology at E18.5 and P0, most AT1 cells in mutant lungs appeared densely packed and failed to flatten out, as observed by PDPN immunostaining (Fig. 1h). The numbers of distal airway epithelial cells including club cells and ciliated cells did not appear to be significantly altered between mutants and wild-type siblings (Supplementary Fig. 1g). Taken together, these results indicate that mutants exhibit disrupted lung epithelial and mesenchymal cell differentiation, resulting in abnormal AT1 cell morphology and defective saccular development.

Identification of a missense mutation in the Myh10 gene. To identify the causative mutation, we carried out whole-exome sequencing of mutant and wild-type siblings and identified a missense Leu-to-Arg mutation (c.T1373G;p.L458R) in the motor domain of NMHC II-B, which is encoded by the Myh10 gene (Figs. 2a, d). Next, we performed genetic linkage analysis by 
a

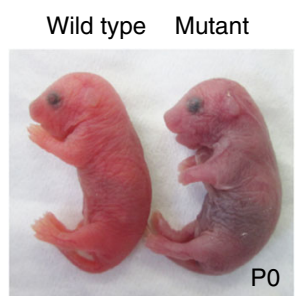

b

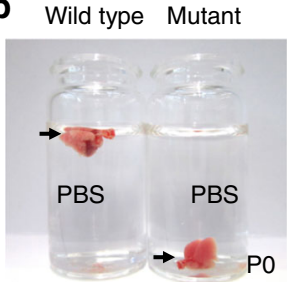

C

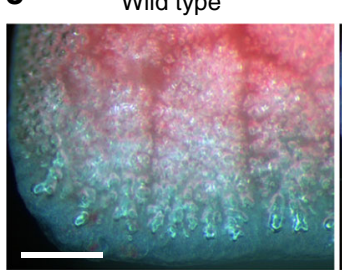

Mutant

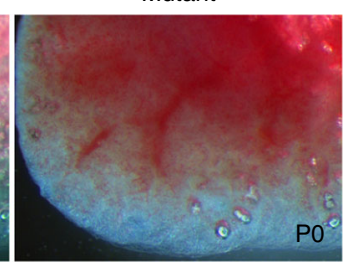

d
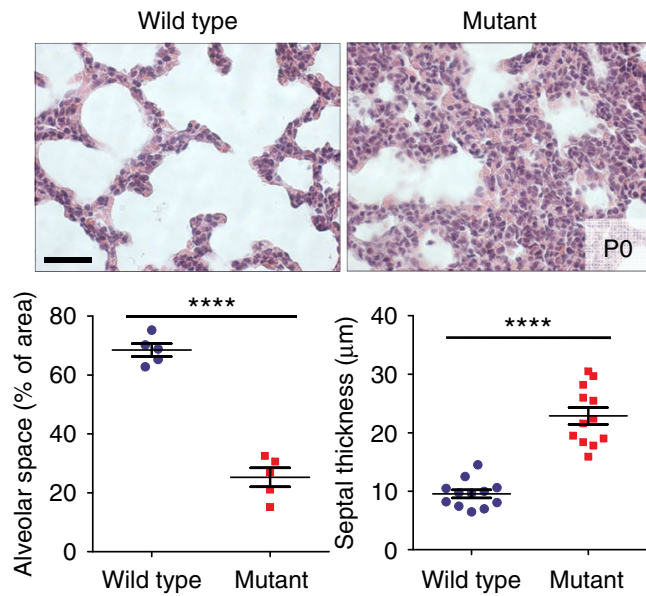

f

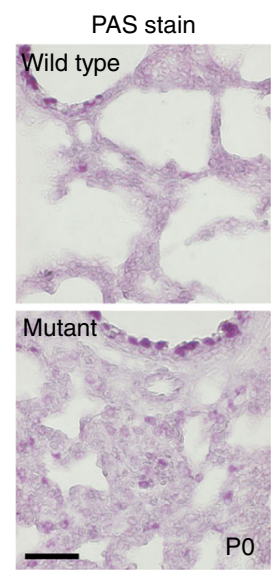

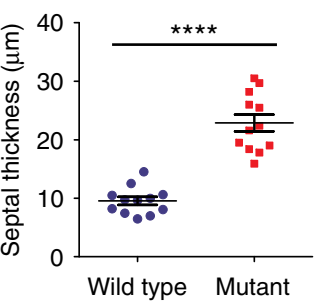

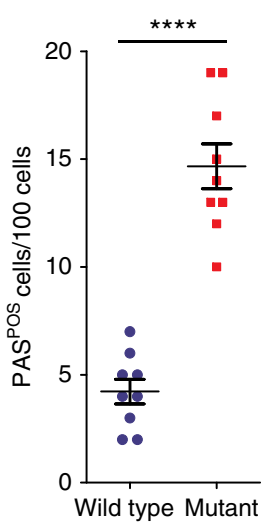

e

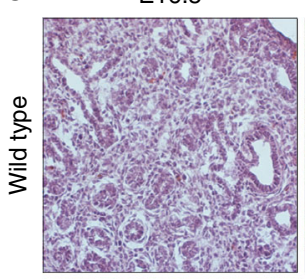

E17.5
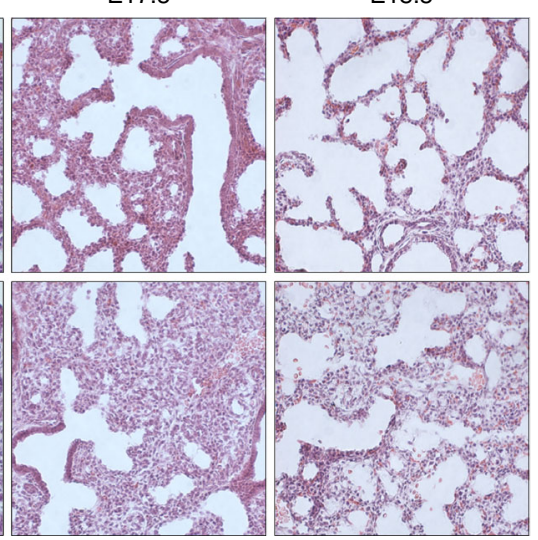

h
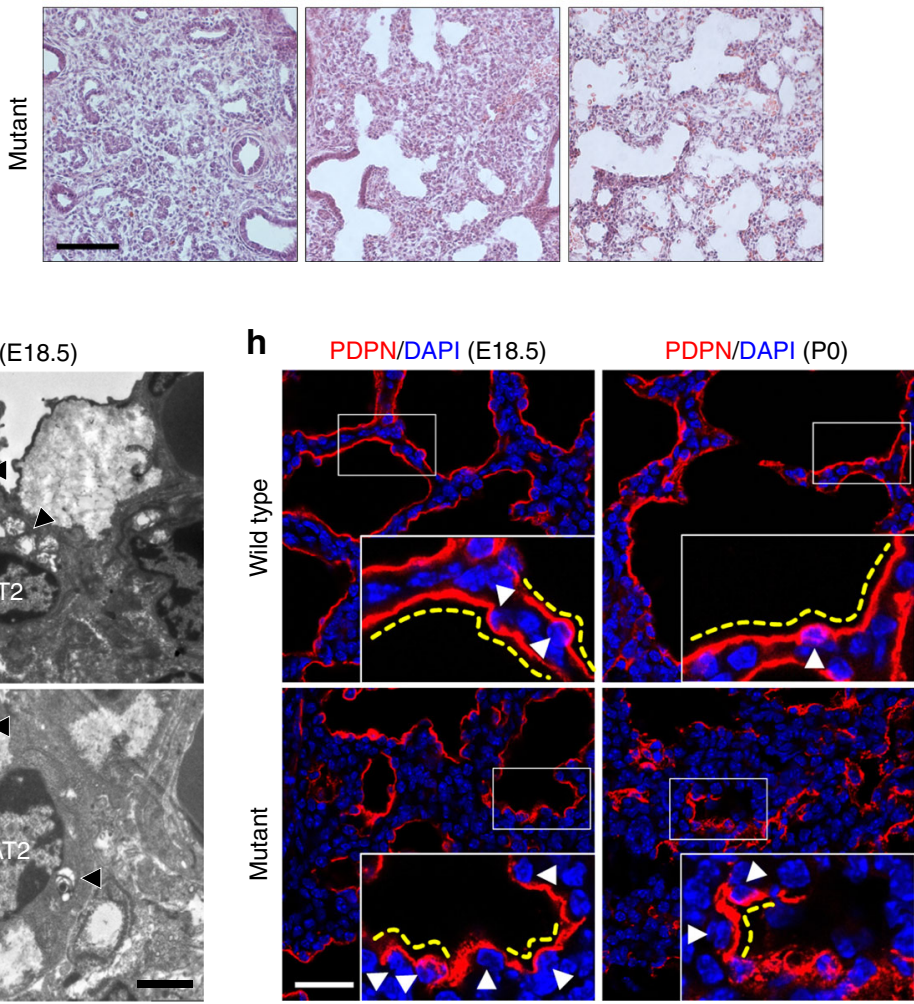

g

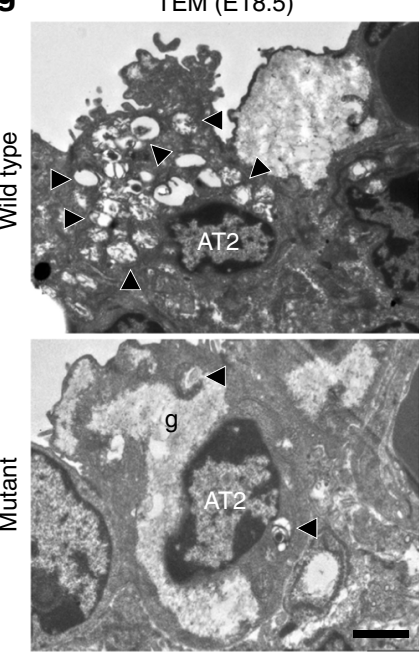

Fig. 1 The ENU-induced mutant exhibits lung development defects. a Gross morphology of wild-type $(n=15)$ and mutant $(n=12)$ PO mice. b Floating assay for wild-type $(n=10)$ and mutant $(n=8)$ PO lungs. c Representative pictures of distal lung from wild-type $(n=10)$ and mutant $(n=8)$ PO mice.

d Hematoxylin and eosin (H\&E) staining and morphometric analysis of alveolar space and septal thickness in wild-type $(n=15)$ and mutant $(n=10)$ PO lungs. e H\&E staining of wild-type ( $n=6$ for each stage) and mutant ( $n=5$ for each stage) lungs at E16.5, E17.5, and E18.5. f PAS staining ( $n=15$ wild types, $n=10$ mutants) and phenotype quantification at PO. $\mathbf{g}$ Representative TEM images of AT2 cells in wild-type $(n=3)$ and mutant $(n=3)$ lungs at E18.5. Arrowheads point to lamellar bodies in AT2 cells; g glycogen. $\mathbf{h}$ Immunostaining for PDPN in wild-type $(n=8$ per each stage) and mutant $(n=8$ per each stage) lungs at E18.5 and PO. Arrowheads point to AT1 nuclei; yellow dotted lines outline AT1 cell morphology. Error bars are means \pm s.e.m. ${ }^{\star \star \star \star} P<$ 0.0001 , two-tailed Student's t-test. Scale bars: $500 \mu \mathrm{m}(\mathbf{c}), 50 \mu \mathrm{m}(\mathbf{d}, \mathbf{e}), 30 \mu \mathrm{m}(\mathbf{f}, \mathbf{h}), 2 \mu \mathrm{m}(\mathbf{g})$

genotyping 102 G4 and G5 mutant mice and found complete association between the lung phenotype and the Myh10 mutation (Figs. 2b, c). We then performed a complementation test between the ENU-induced Myh10 mutant and a Myh10 null allele 22 . Trans-heterozygous mice exhibited the same lung and heart phenotypes as the Myh10 global $^{27,28}$ and ENU-induced mutants, indicating that the loss of Myh10 function is responsible for the lung phenotype (Supplementary Fig. 3a, b). In addition, the localization of MYH10 ${ }^{\mathrm{L} 458 \mathrm{R}}$ was different than that of MYH10 10 T in cultured fibroblasts: while MYH10 ${ }^{\mathrm{WT}}-\mathrm{Myc}$ was localized as scattered punctae in stress fibers and lamellae ${ }^{29}, \mathrm{MYH}_{10} \mathrm{~L}^{\mathrm{L}} 58 \mathrm{R}$ Myc appeared dissociated from the actin bundles of stress fibers (Supplementary Fig. 3c). To quantify the expression levels of Myh10 mRNA and protein in the P0 mutant lungs, we used reverse transcriptase-quantitative PCR (RT-qPCR), western blotting, and immunostaining. Expression of Myh10 mRNA was

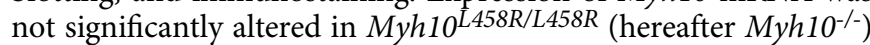
lungs compared with $M y h 10^{+/+}$and $M y h 10^{+/ L 458 R}$ (hereafter $\left.M y h 10^{+/-}\right)$lungs. However, MYH10 protein expression was dramatically reduced in $M y h 10^{-/-}$lungs compared with 


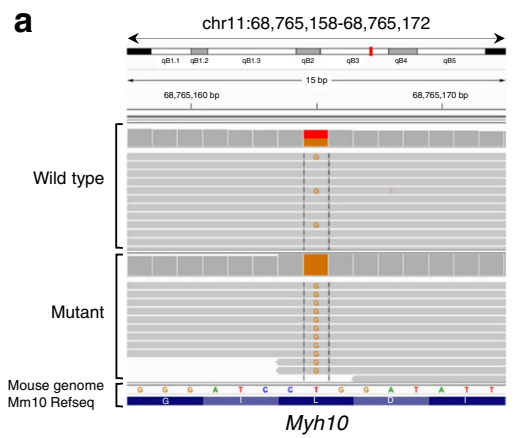

b

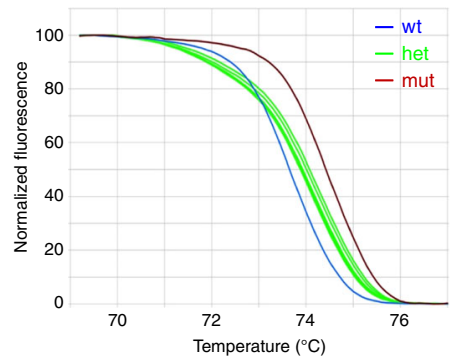

C

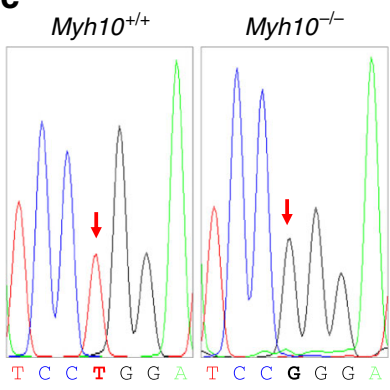

d

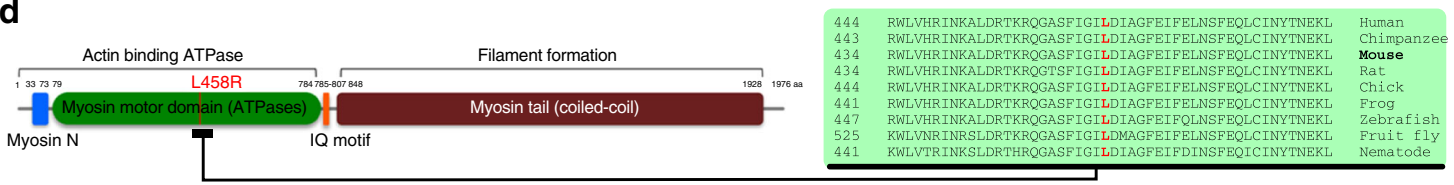

e Myh10

Myh10
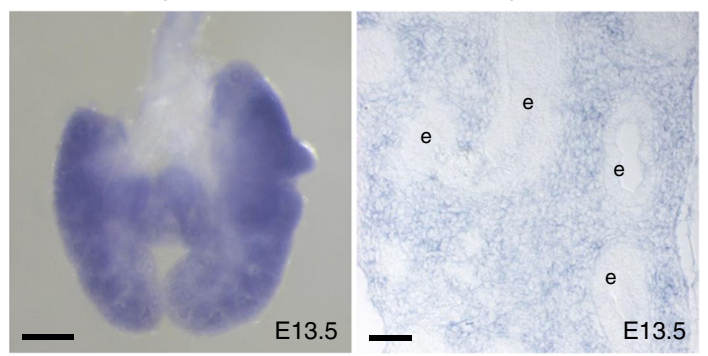

f

MYH10/TbX4/DAPI

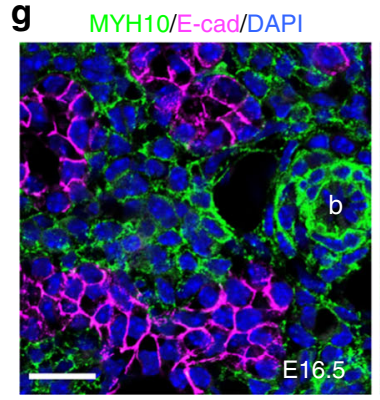

MYH10/ $\alpha-S M A / D A P I$
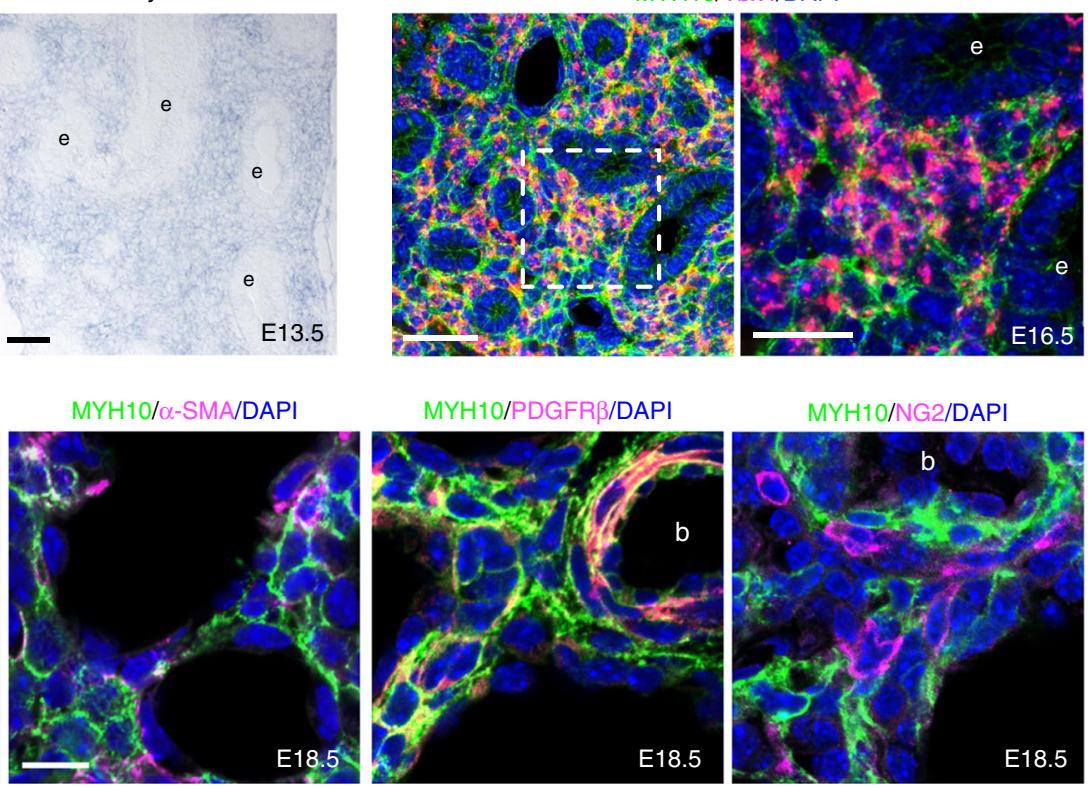

MYH10/NG2/DAPI

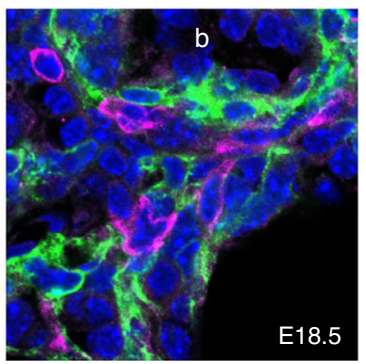

Fig. 2 Isolation of the causative lesion and expression pattern of Myh10. a Integrative genomics viewer (IGV) snapshot of the c.T1373G mutation in Myh10 (chr 11:68,765,165). b Normalized melting curves showing the Myh10 mutation by high-resolution melting analysis (HRMA). c Chromatogram of two different genotypes of Myh10 by Sanger sequencing. $\mathbf{d}$ Schematic diagram of MYH10 protein domains and the relative position of the L458 residue, which is conserved from worms to humans. e In situ hybridization for Myh10 expression in E13.5 whole-mount and on cryosectioned lungs; e epithelium. f Double staining for Tbx4 mRNA (red) and MYH10 protein (green) on E16.5 lung sections; e epithelium. $\mathbf{g}$ Immunostaining for MYH10, E-cadherin (marking epithelial cells), $\alpha$-SMA (marking myofibroblasts and smooth muscle cells), PDGFR- $\beta$ (marking myofibroblasts and smooth muscle cells), and NG2 (marking pericyte-like cells) on E16.5 and E18.5 lung sections; b blood vessel. Scale bars: $200 \mu \mathrm{m}$ (e (left)), $50 \mu \mathrm{m}$ (e (right), f (left)), $20 \mu \mathrm{m}$ (f (right), $\mathbf{g}$ (left)), $10 \mu \mathrm{m}$ (g (right))

Myh10+/+ and Myh10+/- lungs (Supplementary Fig. 3d). These data suggest that possibly due to their inability to bind actin bundles, MYH10 $10^{\mathrm{L} 458 \mathrm{R}}$ proteins are highly unstable and thus subsequently degraded. To test the expression pattern of Myh10 in the developing lung, we performed in situ hybridization and immunostaining. At E13.5, Myh10 transcripts were specifically detected in mesenchymal tissue in both whole-mount and cryosectioned lungs (Fig. 2e). At E16.5, Tbx4-positive mesenchymal cells $^{30}$ co-expressed MYH10, whereas E-cad-positive epithelial cells did not (Figs. 2f, g). At E18.5, MYH10 expression colocalized with $\alpha$-smooth muscle actin ( $\alpha$-SMA) and platelet derived growth factor receptor-beta (PDGFR- $\beta$ ) expression in the lung mesenchyme, but not with NG2, VE-cadherin, or PDPN expression (Fig. 2g, Supplementary Fig. 3e). These results indicate that in developing lungs, Myh10 is specifically expressed in mesenchymal cells including myofibroblasts, lipofibroblasts, and smooth muscle cells but not in endothelial cells, pericyte-like cells, or epithelial cells.

Myh10 deficiency causes defects in ECM remodeling. To understand the molecular mechanisms of Myh10 function during lung development, we analyzed the transcriptome of $M y h 10^{+/+}$ and $M y h 10^{-/-}$lungs at the saccular stage. Many of the genes downregulated in $\mathrm{Myh} 10^{-/-}$lungs are known to participate in cell adhesion and contraction, as well as ECM remodeling 
a

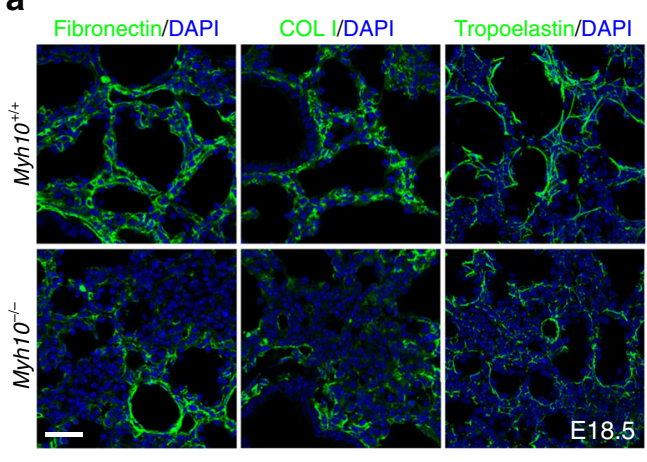

d
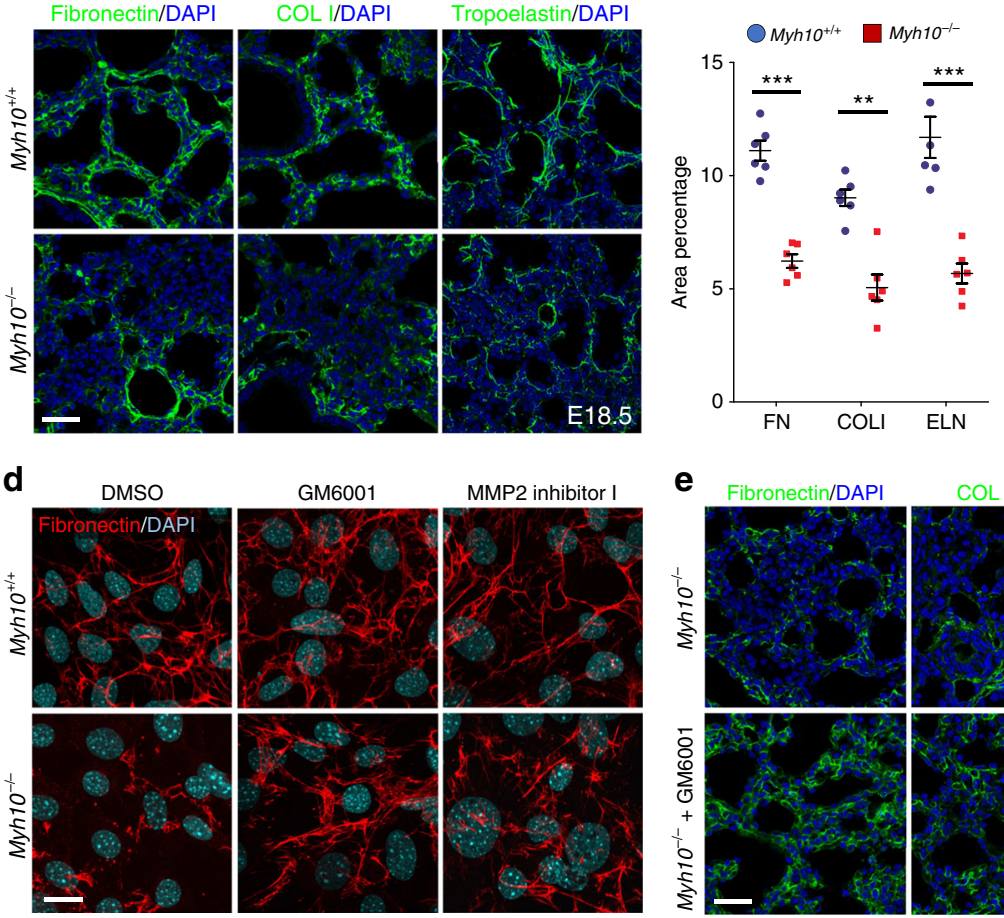

b

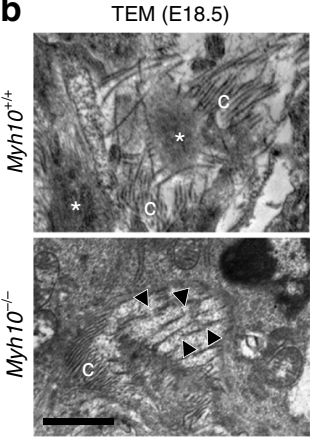

c

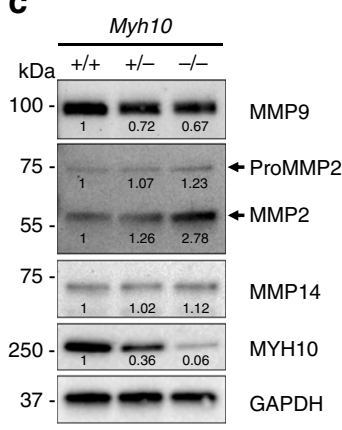

e
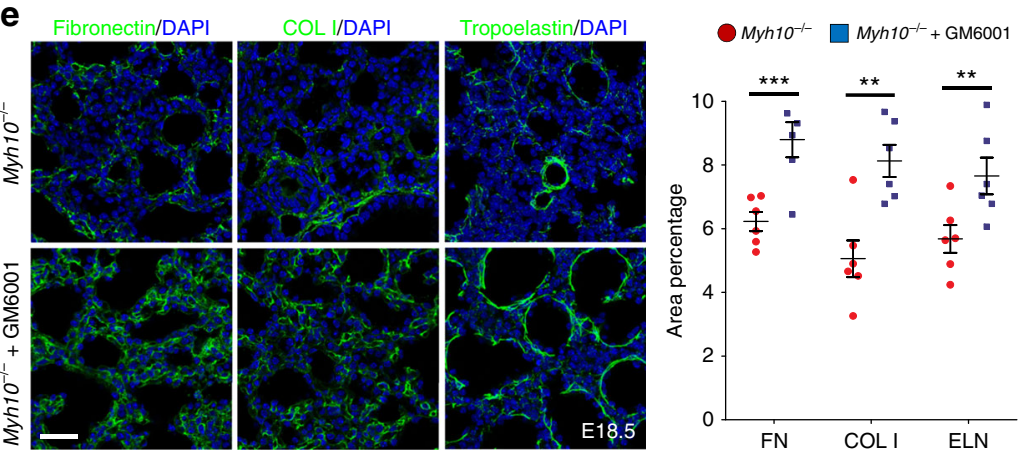

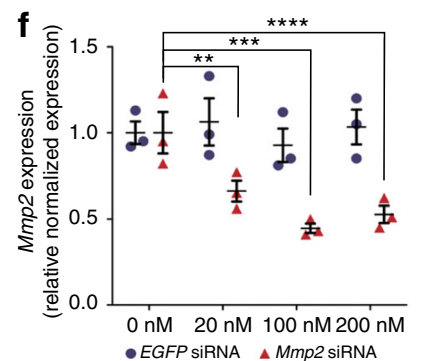

g
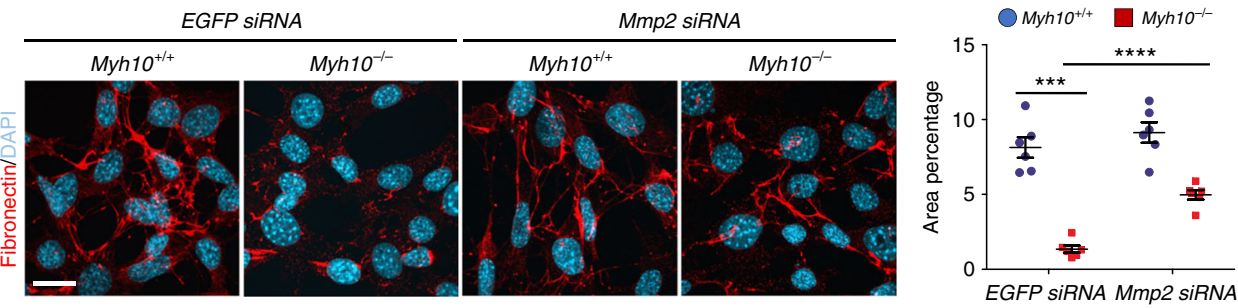

Fig. 3 Myh10 deficiency leads to disrupted ECM remodeling. a Immunostaining and quantification for Fibronectin, type I collagen (COL I), and Tropoelastin on Myh10+/+ $(n=10)$ and Myh10 $/-(n=10)$ E18.5 lung sections. b Representative TEM images comparing elastic fibers in Myh10 $/+(n=3)$ and Myh10 $/-$ $(n=3)$ E18.5 lungs. Asterisks and arrowheads point to bundles and fragmented bundles of elastic fibers, respectively; c collagenous fibrils. $\mathbf{c}$ Representative western blot (from three individual sets of lung lysates) of Myh10+/+, Myh10+/-, and Myh10\%- lung lysates at E18.5. Values represent the densitometric ratio of $\mathrm{Myh10}^{+/-}$and $\mathrm{Myh}^{-/-}$to $\mathrm{Myh10}^{+/+}$after normalization to GAPDH. d Immunostaining (from five individual sets of cultured cells) for Fibronectin in DMSO, GM6001, or MMP2 inhibitor I-treated $\mathrm{Myh}^{+/ /+}$and Myh10/- embryonic fibroblasts. e Immunostaining and quantification for Fibronectin, COL I, and Tropoelastin on Myh10\%- $(n=5)$ and GM6001-injected Myh10\% $(n=5)$ E18.5 lung sections. $\mathbf{f}$ Expression level of Mmp2 in EGFP siRNA and Mmp2 siRNA-treated Myh10+/+ embryonic fibroblasts (from three replicates and individual sets of cultured cells) by RT-qPCR. $\mathbf{g}$ Immunostaining and quantification for Fibronectin in EGFP siRNA and Mmp2 siRNA-treated Myh10+/+ and $\mathrm{Myh10}^{-/-}$embryonic fibroblasts. Error bars are means \pm s.e.m. ${ }^{\star \star} P<0.01,{ }^{\star \star \star} P<0.001,{ }^{\star \star \star \star} P<0.0001$, two-tailed Student's $t$-test. Scale bars: $30 \mu \mathrm{m}(\mathbf{a}, \mathbf{e}), 20 \mu \mathrm{m}(\mathbf{d}, \mathbf{g}), 500 \mathrm{~nm}(\mathbf{b})$

(Supplementary Fig. 4a, Supplementary Data 1), prompting us to investigate the roles of NM II/MYH10 in this process. First, we examined the expression levels of several ECM components. Immunostaining and western blot data showed that expression of Fibronectin (FN), type I Collagen (COL I), and Tropoelastin, which form the interstitial $\mathrm{ECM}^{31}$, appeared significantly reduced in E18.5 Myh10 $1-$ lungs (Fig. 3a, Supplementary Fig. 4b). In contrast, expression of basement membrane (BM) ECM components $^{31}$ type II (COL II) and type IV Collagen (COL IV), as well as that of Laminin, were not altered, suggesting that defects in MYH10 function affect the expression of proteins found in the interstitial ECM of alveoli but not of those in the alveolar BM (Supplementary Fig. 4d). However, RNA expression of FN, Col I, and Tropoelastin was unaltered in E18.5 Myh10/- lungs compared with wild-type lungs (Supplementary Fig. 4c). Using TEM, we analyzed ECM organization in the alveolar interstitium at
E18.5. Mature elastic fibers were largely absent in the ECM of Myh $10^{-/}$lungs, and elastic bundles appeared sparse and fragmented compared with the wild-type pattern (Fig. 3b). These data suggest that Myh10 deficiency disrupts expression levels and localization of ECM components, including FN, Col I, and Elastin. MYH10 is known to mediate primary cilia formation in a human retinal pigmented epithelial (RPE1) cell line ${ }^{32,33}$. To test whether MYH10 regulates cilia formation in embryonic fibroblasts, we performed immunostaining for anti-acetylated $\alpha$ tubulin to detect primary cilia. We found no obvious defects in cilia formation in Myh10-deficient fibroblasts compared with wild-type fibroblasts (Supplementary Fig. 4e). Moreover, expression of components of the Wnt, Sonic hedgehog (Shh), and fibroblast growth factor signaling pathways, which are involved in lung cell proliferation and differentiation ${ }^{4,34}$, was not altered in $M y h 10^{-/-}$lungs compared with wild-type siblings (Supplementary 
a Selected enriched Gene Ontology terms

Downregulated proteins

\begin{tabular}{|c|l|c|r|}
\hline Terms & \multicolumn{1}{|c|}{ Category } & $\begin{array}{c}\text { No. of } \\
\text { proteins }\end{array}$ & \multicolumn{1}{c|}{$p$-value } \\
\hline GO:0032103 & Positive regulation of response to external stimulus & 25 & $1.09 \mathrm{E}-07$ \\
\hline GO:0030199 & Collagen fibril organization & 12 & $1.09 \mathrm{E}-07$ \\
\hline GO:0009605 & Response to external stimulus & 110 & $9.27 \mathrm{E}-06$ \\
\hline GO:0030198 & Extracellular matrix organization & 24 & 0.000124 \\
\hline GO:0009611 & Response to wounding & 38 & 0.000124 \\
\hline GO:0002682 & Regulation of immune system process & 72 & 0.000146 \\
\hline GO:0032101 & Regulation of response to external stimulus & 47 & 0.000299 \\
\hline GO:0006935 & Chemotaxis & 32 & 0.00030 \\
\hline GO:0048646 & $\begin{array}{l}\text { Anatomical structure formation involved in } \\
\text { morphogenesis }\end{array}$ & 79 & 0.000871 \\
\hline GO:0048584 & Positive regulation of response to external stimulus & 106 & 0.001184 \\
\hline GO:0001501 & Skeletal system development & 33 & 0.001287 \\
\hline GO:0072358 & Cardiovascular system development & 52 & 0.001332 \\
\hline GO:0022610 & Biological adhesion & 104 & 0.002075 \\
\hline GO:0043065 & Positive regulation of apoptotic process & 50 & 0.002076 \\
\hline
\end{tabular}

b

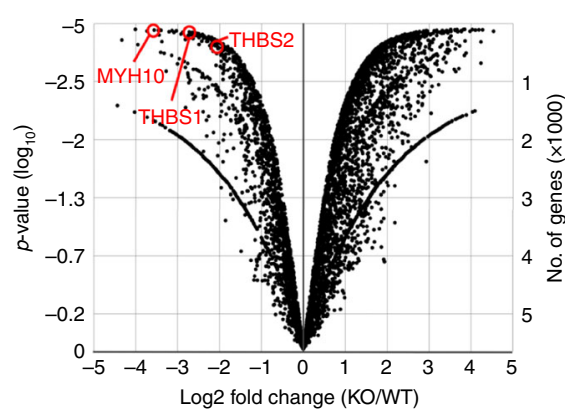

C

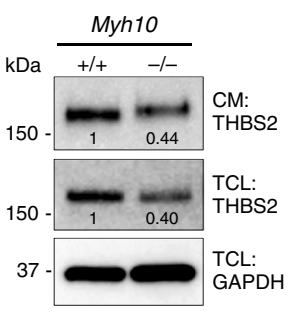

Upregulated proteins

\begin{tabular}{|c|l|c|c|}
\hline Terms & \multicolumn{1}{|c|}{ Category } & $\begin{array}{c}\text { No. of } \\
\text { proteins }\end{array}$ & $p$-value \\
\hline GO:0009127 & $\begin{array}{l}\text { Purine nucleoside monophosphate biosynthetic } \\
\text { process }\end{array}$ & 28 & 0.000169 \\
\hline GO:0007010 & Cytoskeleton organization & 202 & 0.000445 \\
\hline GO:0044763 & Single-organism cellular process & 1280 & 0.000500 \\
\hline GO:0051186 & Cofactor metabolic process & 87 & 0.000914 \\
\hline GO:0045445 & Myoblast differentiation & 11 & 0.001907 \\
\hline GO:0032989 & Cellular component morphogenesis & 178 & 0.002225 \\
\hline GO:0030856 & Regulation of epithelial cell differentiation & 18 & 0.003129 \\
\hline GO:0032507 & Maintenance of protein location in cell & 26 & 0.005557 \\
\hline GO:0044711 & Single-organism biosynthetic process & 173 & 0.005734 \\
\hline GO:0022604 & Regulation of cell morphogenesis & 85 & 0.006341 \\
\hline GO:0022603 & $\begin{array}{l}\text { Regulation of anatomical structure } \\
\text { morphogenesis }\end{array}$ & 134 & 0.007935 \\
\hline GO:0048858 & Cell projection morphogenesis & 106 & 0.009687 \\
\hline GO:0009653 & Anatomical structure morphogenesis & 309 & 0.015052 \\
\hline GO:0048869 & Cellular developmental process & 471 & 0.015085 \\
\hline & & & \\
\hline
\end{tabular}

d
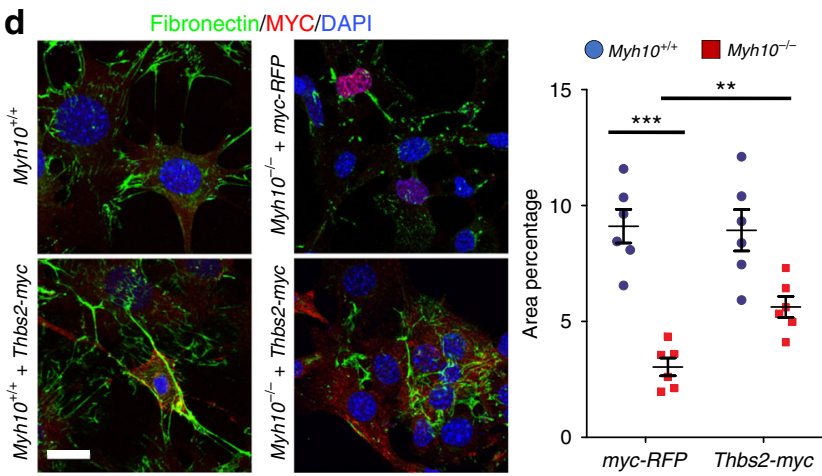

Fig. 4 MYH10 modulates MMP activity by regulating THBS. a GO classification (all components with $P$-value $<0.05$ ) of differentially expressed proteins

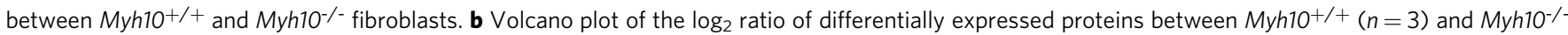
$(n=3)$ fibroblasts. c Representative western blot (from three individual sets of cultured cells) for THBS2 from Myh10 ${ }^{+/+}$and Myh10 $/-$fibroblasts. Values represent the densitometric ratio of $\mathrm{Myh10}^{-/-}$to $\mathrm{Myh}_{10} \mathrm{H}^{+}+$after normalization to GAPDH. CM, conditioned medium; TCL total cell lysate.

d Immunostaining and quantification (from four individual sets of cultured cells) for fibronectin and Myc epitope of $M y$ h10 $/ /+$ and $M y h 10^{-/-}$fibroblasts transfected with myc-RFP or Thbs2-myc. Error bars are means \pm s.e.m. ${ }^{\star \star} P<0.01$, ${ }^{\star \star \star} P<0.001$, two-tailed Student's $t$-test. Scale bar: $20 \mu \mathrm{m}$ (d)

Fig. 4f), indicating that these signaling pathways are not responsible for the phenotypes observed in Myh10-deficient fibroblasts and lungs.

Excess MMP2 activity causes defective ECM remodeling. Metalloproteinases are a group of proteins essential for ECM remodeling, which includes members of the matrix metalloproteinase (MMP) and a disintegrin and metalloproteinase with thrombospondin motifs (ADAMTS) family members ${ }^{35-37}$. During embryonic development, MMP2 is one of the most highly expressed metalloproteinases in the lung ${ }^{38}$. Through both MMP activity assays and western blotting, we found a dramatic increase of MMP2 activity in lysates of E18.5 $\mathrm{Myh}^{-/-}$lungs and of $M y h 10^{-/-}$cultured fibroblasts compared with wild-type samples (Fig. 3c, Supplementary Fig. 5a, b). To test whether MMP inhibition could rescue the Myh10 mutant phenotypes, we first treated wild-type and Myh10 mutant embryonic fibroblasts ${ }^{39}$ with GM6001, a broad-spectrum MMP inhibitor ${ }^{40}$, and with the MMP2-specific inhibitor I ${ }^{41}$. Wild-type fibroblasts displayed wellorganized, linear FN fibers; in contrast, only small aggregates of FN marked the cell surface of Myh10-deficient fibroblasts. Interestingly, the FN matrix defect was partially rescued by treatment with GM6001 or MMP2 inhibitor I (Fig. 3d, Supplementary Fig. 5c). Next, we administered GM6001 to pregnant females daily, covering a developmental time window from E16.5 to E18.5. This regimen was sufficient to partially rescue defective ECM remodeling in $M y h 10^{-/-}$lungs (Fig. 3e). In addition, the FN matrix defect in $M y h 10^{-}$-fibroblasts was partially rescued by small interfering RNA (siRNA)-mediated Mmp2 knock-down (Figs. 3f, g). To investigate at which specific step Myh10 regulates ECM production, we assessed the expression of elastin assembly components in Myh10-deficient lungs. The expression of Fibrillin-1/Fbn1, Fibrillin-2/Fbn2, Fibulin-4/Fbln4, Fibulin-5/Fbln5, Lox, and Loxl1 $1^{42,43}$ was not significantly changed in E18.5 Myh10/- lungs compared with wild-type lungs (Supplementary Fig. 5d). Moreover, Myh10 deficiency did not cause defects in FN assembly in fibroblasts (Supplementary Fig. 5e), altogether suggesting that Myh10 does not directly control the expression, crosslinking, or assembly of ECM components in fibroblasts or developing lungs. Collectively, these results suggest that Myh10 deficiency leads to increased MMP activity, which disrupts ECM remodeling in fibroblasts in vitro and in developing lungs in vivo.

To understand how MYH10 regulates MMP activation, we performed proteomic profiling of wild-type and mutant fibroblasts and detected lower levels of Thrombospondins (THBS1 and THBS2) in $\mathrm{Myh}^{-/} \mathrm{O}^{-}$fibroblasts (Figs. 4a, b, Supplementary Data 2). THBSs are matricellular proteins that interact with MMPs to inhibit their activities ${ }^{44-46}$. Consistently, we found a dramatic decrease in THBS1 and THBS2 expression levels in cell lysates and conditioned media of mutant fibroblasts as well as in 
a

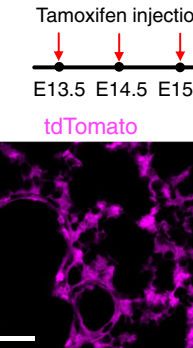

b

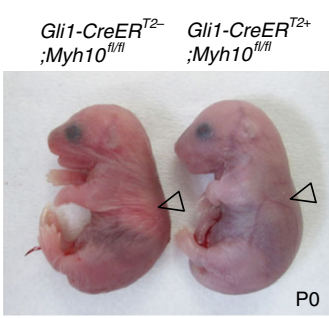

C

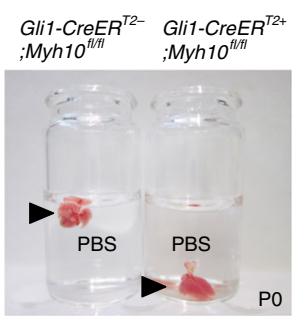

d
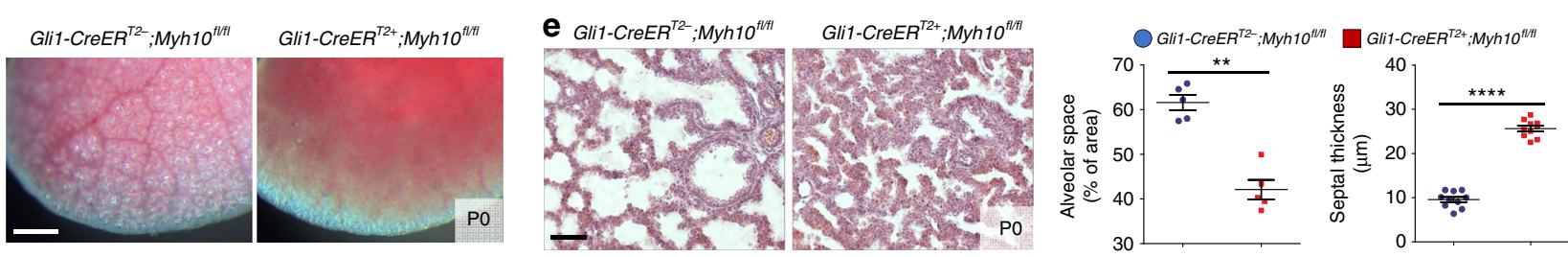

$\mathbf{f}$

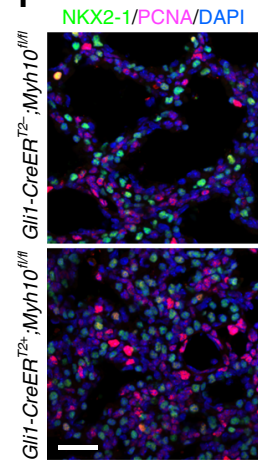

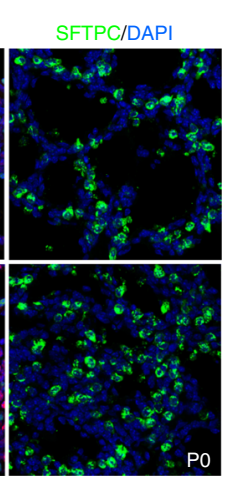
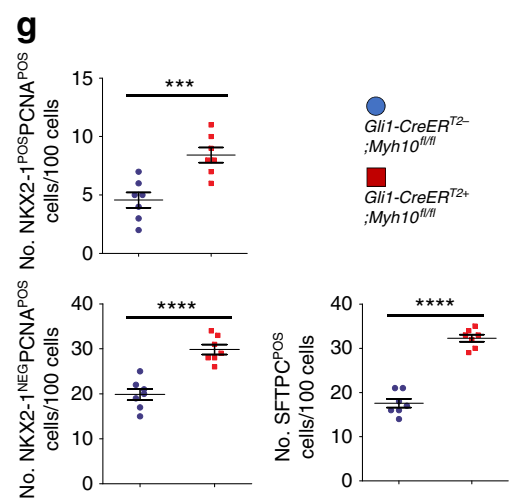

h
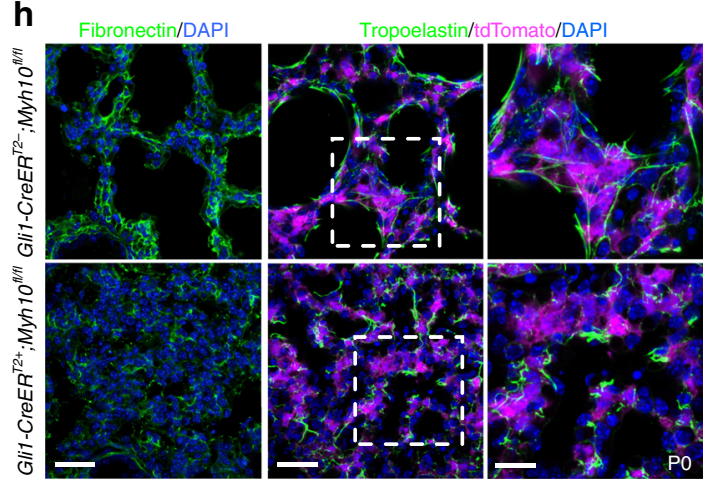

Fig. 5 Mesenchyme-specific Myh10 deletion disrupts lung development. a Diagram indicating time points of tamoxifen injections and tissue collection. Immunostaining for E-cadherin in GliT-CreERT2;ROSA26tdTomato PO lungs. b Gross morphology of wild-type $(n=10)$ and GliT-Myh10cKO ( $n=8)$ PO mice. Arrowheads point to inflated (left) and non-inflated lungs (right). c Floating assay for wild-type $(n=10)$ and Glit-Myh10cKO ( $n=8)$ PO lungs.

d Representative pictures of distal lung from wild-type $(n=10)$ and Gli1-Myh10cKO $(n=8)$ PO mice. e H\&E staining and morphometric analysis of alveolar space and septal thickness in wild-type $(n=10)$ and Glit-Myh10CKO $(n=8)$ lungs at PO. $\mathbf{f}$ Immunostaining for NKX2-1, PCNA, and SFTPC in wild-type $(n=$ $10)$ and Glit-Myh10'KO $(n=8)$ PO lungs. $\mathbf{g}$ Quantification of cell proliferation and differentiation in wild-type and GliT-Myh10cKO PO lungs. $\mathbf{h}$ Immunostaining for fibronectin, tropoelastin, and tdTomato in wild-type $(n=10)$ and Gli1-Myh10cKO $(n=8)$ PO lungs. Error bars are means \pm s.e.m. ${ }^{\star \star} P<0.01$, ${ }^{\star \star \star} P<0.001$, ${ }^{\star \star \star \star} P<0.0001$, two-tailed Student's t-test. Scale bars: $500 \mu \mathrm{m}$ (d), $50 \mu \mathrm{m}$ (e), $30 \mu \mathrm{m}$ (a, f, h (left, middle)), $15 \mu \mathrm{m}$ (h (right))

Myh10/- lungs (Fig. 4c, Supplementary Fig. 5f, g), further validating our proteomics results. Importantly, overexpression of

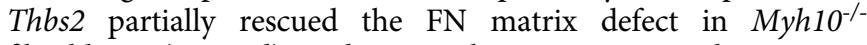
fibroblasts (Fig. 4d), indicating that MYH10 regulates MMP activation through THBS.

Myh10 function is required in lung mesenchymal cells. To determine which cells require Myh10 function during mouse lung development, we utilized a floxed $M y h 10^{f l / f l} \operatorname{lin} e^{47}$. First, we deleted Myh10 expression in lung epithelial or endothelial cells by using ShhCre or Tek-CreER ${ }^{T 2}$, respectively, and found no obvious breathing dysfunction or lung defects (Supplementary Fig. 6a-g). To test Myh10 function in the lung mesenchyme, we crossed Gli1-CreER ${ }^{T 2}$

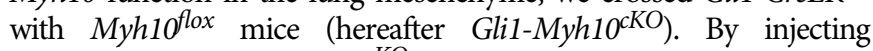
tamoxifen into Gli1-Myh10cKO;tdTomato mice at E13.5-E15.5, we found that Gli1-positive cells did not overlap with E-cad-expressing epithelial cells in P0 lungs (Fig. 5a). Gli1-Myh10 ${ }^{c K O}$ newborn pups exhibited respiratory distress and cyanosis (Fig. 5b). Floating lung assays from wild-type and Gli1-Myh10cKO newborns showed that the mutant lungs failed to inflate with air at birth (Figs. 5c, d). The expression of Myh10 mRNA and protein was dramatically decreased in Gli1-Myh10cKO lungs as compared with wild-type and heterozygous littermates (Supplementary Fig. 7a, b). Gli1-Myh10 $0^{c K O}$ mice also exhibited alveolar collapse, with no apparent cardiac defects (Fig. 5e, Supplementary Fig. 7c). Gli1-Myh10cKO lungs exhibited an increased number of proliferating epithelial (NKX2-1/PCNA double positive) and mesenchymal (NKX2-1-negative/PCNA-positive) cells, as well as an increased number of AT2 cells (SFTPC-positive), as clearly in the ENU-induced allele (Figs. 5f, g). Finally, Gli1-Myh10 cKO lungs exhibited clearly decreased FN staining intensity, as well as truncated and disorganized elastin fibers (Fig. 5h). In summary, mesenchyme-specific loss of Myh10 led to lung defects that were observed in the global Myh10 knockout, as well as in the ENUinduced $M y h 10^{L 458 R}$ allele. These results indicate that Myh10 is specifically required in lung mesenchyme to promote ECM remodeling and cell differentiation.

Myh10 is required for lung alveologenesis. As MYH10 is still highly expressed in mesenchymal tissues throughout postnatal and adult stages (Supplementary Fig. 3e), we investigated Myh10 function during lung maturation and homeostasis. To test the role of Myh10 in alveologenesis, we deleted Myh10 in mesenchymal cells at early postnatal stages before secondary septae formation $^{1,2,5}$ (Supplementary Fig. 7d). By injecting tamoxifen into 
a

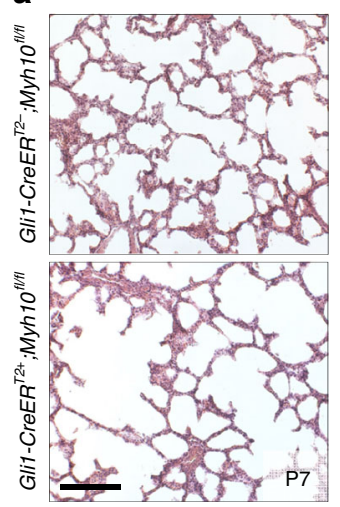

d

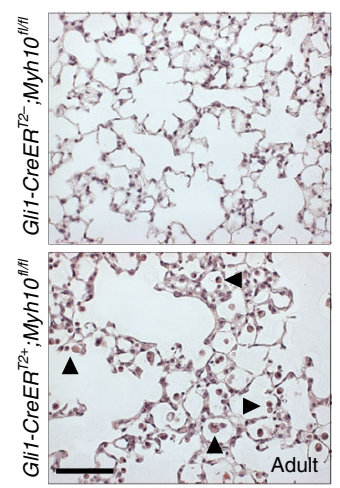

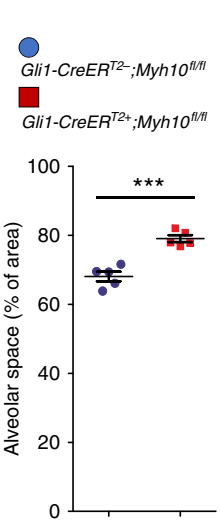
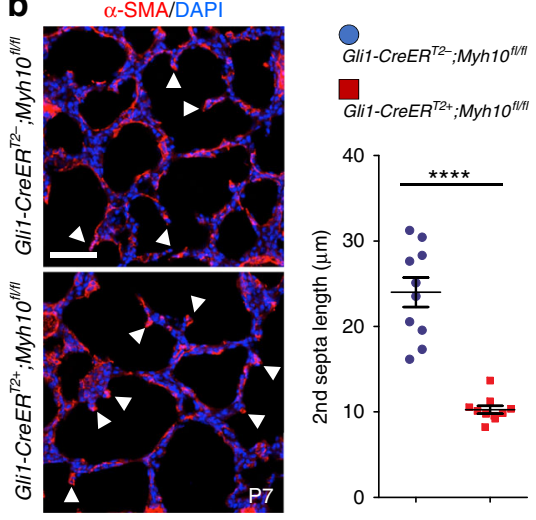

C

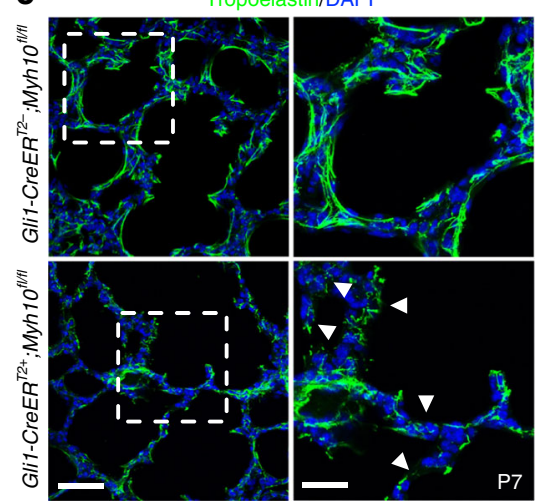

e

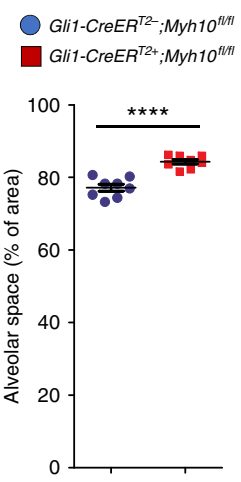

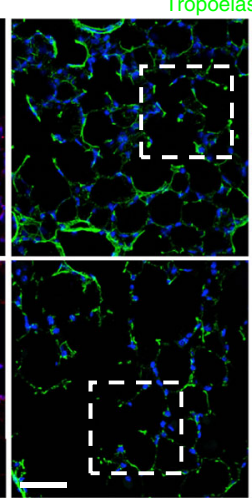

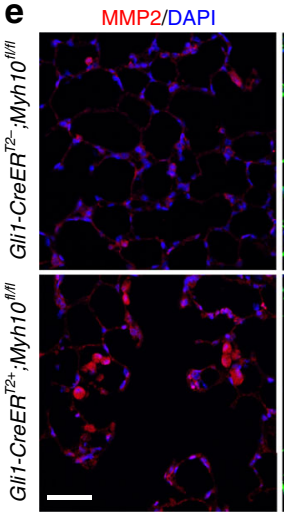

Fibronectin/DAPI

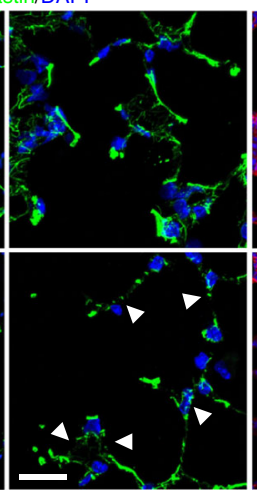

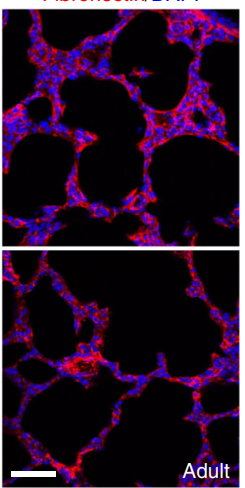

Fig. 6 Myh10 deficiency causes emphysema-like phenotypes. a H\&E staining of wild-type $(n=10)$ and GliT-Myh10cKO $(n=8)$ P7 lungs. Quantification of alveolar space in wild-type and GliT-Myh10cKO P7 lungs. b Immunostaining for $\alpha$-SMA in wild-type $(n=10)$ and Gli1-Myh10cKO ( $n=8$ ) P7 lungs. Arrows point to secondary septae. Quantification of secondary septae length in wild-type and GliT-Myh10CKO P7 lungs. c Immunostaining for Tropoelastin in wildtype $(n=10)$ and GliT-Myh10cKO $(n=8)$ P7 lungs. Arrowheads point to truncated elastin fibers. $\mathbf{d}$ H\&E staining and morphometric analysis of alveolar space in wild-type $(n=8)$ and Glit-Myh10cKO $(n=6)$ adult lungs. Arrowheads point to macrophages. e Immunostaining for MMP2, tropoelastin, and fibronectin in wild-type $(n=8)$ and GliT-Myh10cKO $(n=6)$ adult lungs. Arrowheads point to truncated elastin fibers. Error bars are means \pm s.e.m. ${ }^{\star \star \star} P<0.001,{ }^{\star \star \star \star} P<0.0001$, two-tailed Student's t-test. Scale bars: $50 \mu \mathrm{m}$ (a, d), $30 \mu \mathrm{m}$ (b, c (left), e (left, right)), $15 \mu \mathrm{m}$ (c (right), e (middle))

Gli1-Myh10 ${ }^{c K O}$; tdTomato mice at P0, we found that tdTomatoexpressing cells did not overlap with E-cad-expressing epithelial cells in P7 lungs (Supplementary Fig. 7d-f). Gli1-Myh10 ${ }^{c K O}$ mice displayed a simplified, dilated alveolar structure and shorter secondary septae compared with wild-type siblings (Figs. 6a, b). Furthermore, FN expression was significantly decreased, and elastin fibers appeared disorganized and truncated (Fig. 6c), suggesting that Myh10 is also required for secondary septation through ECM remodeling during postnatal lung development.

To test whether Myh10 participates in lung homeostasis, we deleted Myh10 in the mesenchymal tissue of adult mice (Supplementary Fig. 7g). Gli1-Myh10 ${ }^{c K O}$;tdTomato mice were injected with tamoxifen at 2 months, and tdTomato expression was detected in lung mesenchymal cells 1 month later, consistent with previous observations ${ }^{48}$ (Supplementary Fig. $7 \mathrm{~g}, \mathrm{~h}$ ). At this stage, Gli1-Myh10 $0^{c K O}$ mice exhibited disrupted septae and enlarged alveolar spaces, accompanied by macrophage accumulation in the alveolar interstitium and alveolar spaces (Fig. 6d and Supplementary Fig. 7i). Moreover, slightly increased numbers of apoptotic cells were observed in Gli1-Myh10 ${ }^{c K O}$ lungs compared with wild-type siblings (Supplementary Fig. 7j). The expression of MMP2 was significantly increased in alveolar interstitial tissues, as well as in macrophages in Gli1-Myh10cKO lungs compared with wild-type siblings (Fig. 6e), suggesting that upregulated MMP2 in the alveolar interstitium and in macrophages, as well an increased number of macrophages, might be involved in alveolar simplification of Myh10-deficient adult lungs. Whereas wild-type lungs displayed a well-organized elastin network, Gli1-Myh10 ${ }^{c K O}$ lungs showed decreased elastin deposition and truncated and disorganized elastin fibers (Fig. 6e), suggesting that Myh10 continues to be required to maintain the elastin fiber network and alveolar structure during lung homeostasis.

MYH10 is downregulated in emphysematous lungs. As the conditional knockout phenotype in adult mice is histologically reminiscent of emphysema, we first measured the expression levels of MYH10 protein in human lung samples from healthy donors and emphysema patients. We found significantly downregulated MYH10 expression in lungs of emphysema patients when compared with healthy donor lungs (Figs. 7a, b). Next, we characterized MYH10-expressing cells in human lungs by immunostaining. Similar to MYH10 expression in mouse embryonic and adult lungs, MYH10-expressing human cells did not overlap with NKX2-1-expressing epithelial cells in the alveolar interstitium (Fig. 7c). MYH10 was strongly detected in smooth muscle cells surrounding blood vessels (Fig. 7d). We next analyzed MYH10 expression in human lung samples from 13 patients with emphysema and 7 healthy donors. In healthy lungs, MYH10 was expressed in smooth muscle cells and mesenchymal 
a

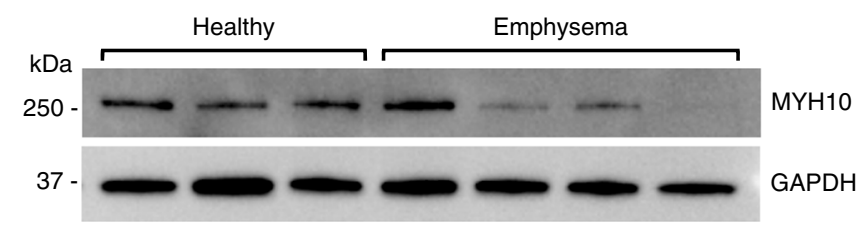

C
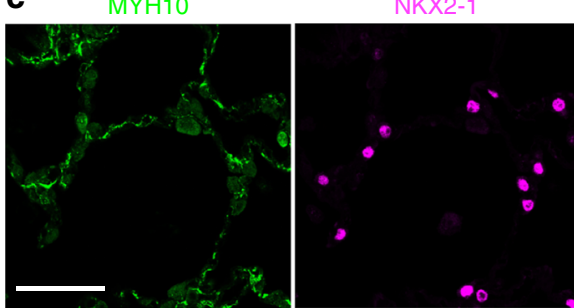

MYH10/NKX2-1/DAPI

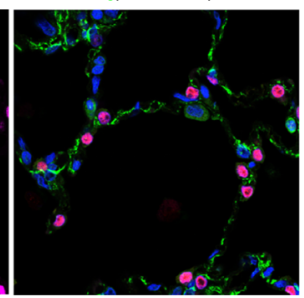

e

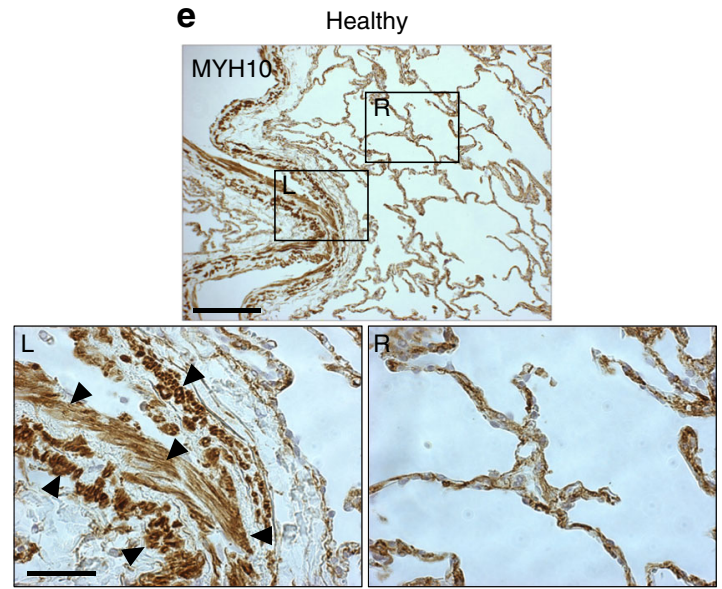

b

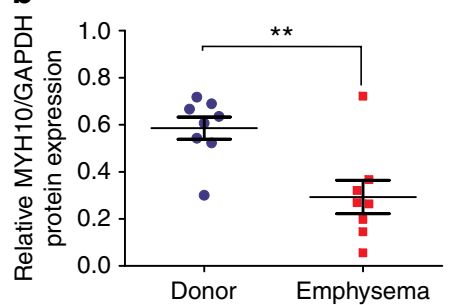

d ${ }_{\mathrm{MYH} 10}$

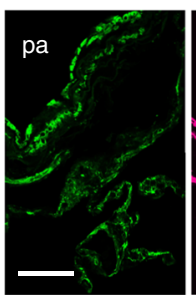

o-SMA
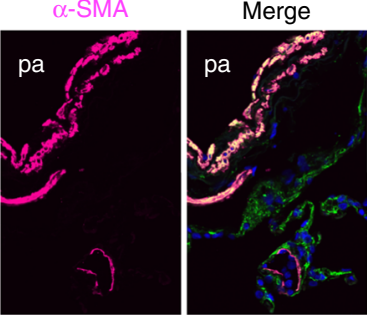

Emphysema

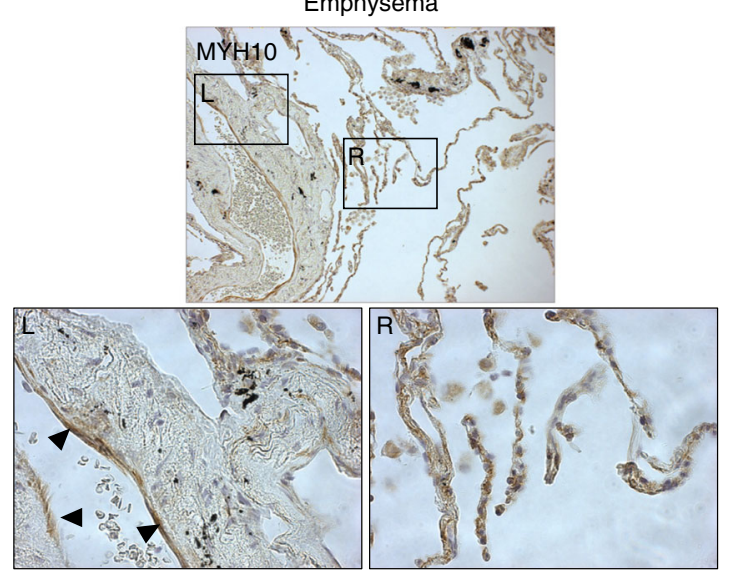

Fig. $7 \mathrm{MYH10}$ is upregulated in emphysematous human lungs. a Representative western blot for human MYH10 of lung tissues from healthy donors (three individuals) and emphysema patients (four individuals). b Quantification of MYH10 expression of lung tissues from healthy donors (three individuals) and emphysema patients (four individuals). c Immunostaining for MYH10 and NKX2-1 in adult human lungs. $\mathbf{d}$ Immunostaining for MYH10 and $\alpha$-SMA in adult human lungs. pa, pulmonary artery. e Immunostaining for MYH10 in healthy control and emphysema patient lungs. Arrowheads point to smooth muscle cells. L left, R right. Error bars are means \pm s.e.m. ${ }^{\star \star} P<0.01$, two-tailed Student's t-test. Scale bars: $100 \mu \mathrm{m}(\mathbf{e}($ top $)), 50 \mu \mathrm{m}(\mathbf{c}, \mathbf{d}), 30 \mu \mathrm{m}(\mathbf{e}($ bottom))

cells of the alveolar interstitium. In contrast, in emphysematous lungs, MYH10 levels were strongly reduced in smooth muscle cells and moderately reduced in the alveolar interstitium (Fig. 7e, Supplementary Fig. 8a, b). Overall, MYH10 was downregulated in $10(77 \%)$ of the 13 emphysematous specimens (Supplementary Table 1). Taken together, these data indicate that MYH10 not only has a critical function in lung alveologenesis, but that it may also play a protective role against the occurrence of emphysema.

\section{Discussion}

Here, we identified a mutation in the mouse Myh10/NM II- $B$ gene through an ENU mutagenesis screen and determined its role during lung development and alveologenesis, through a molecular mechanism involving MMP-mediated ECM remodeling. Myh10 mutant lungs display defects in: (1) ECM remodeling and cell proliferation during the canalicular stage, and (2) distal epithelial cell differentiation, AT1 cell morphogenesis, and interstitial remodeling/tissue morphogenesis during the saccular stage. As a consequence, mutant pups exhibit alveolar collapse and respiratory distress, and die shortly after birth (Supplementary Fig. 9a).

Our results implicate Myh10 in the maintenance of lung ECM components. The ECM is a three-dimensional, dynamic structure that is constantly remodeled to control tissue homeostasis. Dynamic remodeling of the ECM is essential for organ development and homeostasis and its dysregulation can result in pathological conditions. Thus, proteins involved in ECM degradation and modification, including MMPs, are essential for metazoan development ${ }^{35,36}$. ECM-remodeling MMPs are tightly regulated at the transcriptional, post-transcriptional, and posttranslational levels. Examples of biological processes regulating MMP expression and/or function include mechanical forces and actomyosin contractility. Based on our mass spectrometry-based proteomic analysis, we propose a Myosin-dependent regulation of MMP2, which involves another ECM molecule, THBS. Secretion of THBSs and their distribution in the ECM has been shown to be regulated by the actin cytoskeleton. Elevated ECM deposition of THBS1 or THBS2 is associated with increased tissue stiffness, such as in fibrosis and atherosclerotic plaques ${ }^{49,50}$. Interestingly, in these contexts, intracellular tensile forces (contractility) are also increased $^{50}$, suggesting a reciprocal system by which THBS can modulate the actomyosin network. Our results implicate Myh10 in the maintenance of lung ECM components. The structural actomyosin network defects caused by the loss of Myh10 $0^{51,52}$ is likely to inhibit the secretion of THBS, in turn leading to derepression of MMP activity, and disrupted ECM remodeling 
(Supplementary Fig. 9b). Thus, we propose a signaling axis controlling ECM homeostasis through the actomyosin network, THBSs and MMPs. Although our data point to a critical role for MYH10/THBS/MMP signaling in ECM remodeling during lung development and homeostasis, how the actomyosin network regulates extracellular deposition of THBS via MYH10 function will require further investigation.

Our study also uncovers a potential link between loss of MYH10 and COPD. COPD is a common disease worldwide, associated with high morbidity, and characterized by progressive airflow obstruction that is poorly reversible, airway inflammation, and systemic effects ${ }^{15,16}$. The main risk factor for the development of COPD is smoking, but other factors such as air pollutants and genetic determinants have been identified. Pulmonary emphysema, a key symptom of COPD, results from the enzymatic destruction of lung ECM components including elastin and collagen, thereby decreasing tissue stiffness and stability ${ }^{53-55}$. Tissue stiffness has been shown to induce actin cytoskeleton reorganization and contractility, which in turn induces matrix rigidity, and modulates cell adhesion, migration, division, and differentiation ${ }^{56,57}$. In our study, we observed that downregulation of MYH10 expression in human emphysema correlates with disease progression, and Myh10-deficient mice display emphysematous lungs, supporting the notion that Myh10 deficiency might lead to progressive destruction of the lung mesenchyme. Moreover, two genome-wide studies have reported mutations and reduced expression of two actomyosin-related genes, Myosin ID (MYO1D) ${ }^{58}$ and Myosin Light Chain Kinase $(M Y L K)^{59}$ in emphysematous human lungs, thus strengthening the relevance of our model.

Excess MMP activity in lungs is known to be associated with pulmonary diseases including COPD, acute respiratory distress syndrome, sarcoidosis, and tuberculosis ${ }^{60,61}$. In COPD, MMPs are upregulated and their inhibition prevents disease progression in animal models ${ }^{62,63}$. In the present study of Myh10 deficiency during lung development and homeostasis, excess MMP2 activity and consequent defective ECM remodeling partially recapitulate the pathophysiological features of human COPD, especially emphysema. Although mutations in the MYH10 locus have not yet been implicated in the pathogenesis of COPD, further studies are required to investigate possible correlations between NM II/ MYH10 and COPD pathology. Our results from animal models and human patients suggest that structural alterations of the actomyosin network by loss of Myh10 function contribute to the pathogenesis of emphysema. We propose MYH10 as a diagnostic tool for COPD and introduce a mouse model to enable further investigation into the etiology of, and therapeutic approaches for, this disease.

\section{Methods}

Mouse ENU mutagenesis. ENU-injected C57BL/6 males were obtained from Dr. Monica Justice (Baylor College of Medicine, Houston, TX) ${ }^{64}$ and crossed with C57BL/6 females to generate G1 males $(N=170)$. G1 males were subsequently crossed with $\mathrm{C} 57 \mathrm{BL} / 6$ females to generate $\mathrm{G} 2$ females, which were backcrossed to G1 males to generate G3 pups. G3 litters were screened for lung and tracheal malformations from P0 to P7 ${ }^{65}$. Genomic DNA from mutant mice and their littermates was isolated for whole-exome sequencing.

Mouse strains. The following mouse lines were kindly provided for our study: Myh10-flox by Dr. Robert Adelstein; Shh-Cre and Gli1-CreER ${ }^{T 2} ; R_{\text {RSA26 }}$ tdTomato $^{\text {by }}$ Dr. Saverio Bellusci; Tek-CreER ${ }^{T 2}$ by Dr. Stefan Offermanns; and CMV-Cre by Dr. Thomas Braun. Tamoxifen (Sigma) was dissolved in corn oil at $50 \mathrm{mg} / \mathrm{ml}$ and intraperitoneally injected at the indicated developmental stages and frequencies. GM6001 (TOCRIS, catalog no.: 2983) was dissolved in dimethylsulfoxide (DMSO), and fresh dilutions in saline to a total volume of $200 \mu \mathrm{l}$ were prepared immediately before administration to pregnant females. Intraperitoneal injection of GM6001 $(100 \mathrm{mg} / \mathrm{kg}$ ) was performed once daily from E16.5 and lung tissues were collected from E18.5 embryos (Fig. 3e). All animal care and use procedures in this study were approved by the local animal ethics committee (Regierungspräsidium
Darmstadt, Hessen, Germany). All animal studies have been carried out in adherence to the relevant ethical guidelines.

Whole-exome sequencing and genotyping. For whole-exome sequencing, genomic DNA samples were extracted from two wild-type and two mutant pups according to standard protocols. The whole-exome sequencing was performed by the Beijing Genomics Institute (BGI). Genomic DNA was captured using Agilent SureSelect Biotinylated RNA Library (BAITS) and loaded on a HiSeq2000 platform for sequencing. Sequence reads were aligned to the C57BL/6J mouse reference genome (mm10) and analyzed using CLCBio Genomic Workbench and GATK software. Single-nucleotide polymorphisms (SNPs) were called using the GATK haplotype caller, closely following the GATK best practices. Briefly, aligned reads were de-duplicated and re-aligned to all exonic sequences from the Gencode genome annotation (version vM4), taking into account known variants from DBsnp. After base re-calibration, SNP calling was performed within exons, allowing a padding of $100 \mathrm{bp}$ into flanking introns. Variant calls were annotated to known SNPs from DBsnp (version 142) and the functional relevance of variant calls was predicted using Annovar. Next, calls from wild-type mice were merged (unified), while calls from mutant mice were intersected. Intersecting calls were classified as mutant/homozygous if the corresponding wild-type call was heterozygous/absent or as mutant/heterozygous if there was no corresponding wild-type call. For genotyping, an Eco Real-Time PCR System (Illumina) was used for PCR reactions and high-resolution melt analysis. PCR reaction conditions were as follows: $95^{\circ} \mathrm{C}$ for $15 \mathrm{~s}, 40$ cycles of $95^{\circ} \mathrm{C}$ for $2 \mathrm{~s}, 60^{\circ} \mathrm{C}$ for $2 \mathrm{~s}$, and $72{ }^{\circ} \mathrm{C}$ for $2 \mathrm{~s}$. Following PCR, a high-resolution melt curve was generated by collecting SYBRgreen fluorescence data in the $65-95^{\circ} \mathrm{C}$ range. The analyses were performed on normalized derivative plots. The following primers were used: Myh10 (exon 12) forward 5'-TGGATAGGACCAAACGCCAG-3' and Myh10 (exon 12) reverse 5'GGAGAGAGTTGAGGAATACCTC-3'.

Plasmids. Total RNA and complementary DNA (cDNA) were obtained from E13.5 lungs using TRIzol reagent (Life Technologies) and Superscript III reverse transcriptase (Life Technologies), respectively, according to manufacturer's instructions. cDNA encoding the MYH10 (NCBI Acc. No. NM_175260) wild-type and $\mathrm{MYH} 10^{\mathrm{L} 458 \mathrm{R}}$ proteins was PCR amplified using total $\mathrm{CDNA}$ from E13.5 lungs as a template. PCR fragments were cloned into the pcDNA3.1-Myc-His vector at the HindIII site using the Cold Fusion Cloning Kit (System Biosciences). Thbs2 (NCBI Acc. No. NM_011581) cDNA was PCR amplified using total cDNA from E18.5 lungs as a template. PCR fragments were ligated to the pcDNA3.1-Myc-His vector at XhoI/EcoRI sites. The following primers were used: Myh10 forward 5'-TCCGAGCTCGGTACCACTGTTTACAATGGCCCAGAG-3' and Myh10 reverse 5'-TTGTTCGGGCCCAAGCTCTGATTGGGGTGGCTGTG-3'; Thbs2 forward 5'-AGGTCGACGTCACAGGTGGAGACAAGATG-3' and Thbs 2 reverse 5'-TGGAATTCTCGAGGCATCTCTGCACTCA-3'. To synthesize RNA probes for in situ hybridization, cDNA was PCR amplified using total cDNA from E13.5 lungs as a template. PCR fragments were cloned into the pGEM T-easy vector (Promega). The following primers were used: Myh10 forward 5'-

GTACAGAAAGCCCAGACCAAAGA-3' and $M y h 10$ reverse $5^{\prime}$-TTCTTCAT CAGCCACTCATCTGC-3'; Tbx4 forward 5'-CTTCTACCACTGCCTGAAGC GT-3' and Tbx4 reverse 5'-AGTCTCGTCATCCATCGGTCCA-3'.

In situ hybridization and immunohistochemistry. In situ hybridization was performed according to standard procedures. Digoxigenin-labeled RNA probes for Myh10 and Tbx4 (NCBI Acc. No. NM_011536) were used. Hybridization and probe washes were performed at $70^{\circ} \mathrm{C}$. Immunohistochemistry was performed according to a standard protocol. The following antibodies and dilutions were used for immunostaining: acetylated $\alpha$-tubulin (Sigma, 1:5000), CGRP (Abcam, 1:5000), cleaved caspase 3 (Cell Signaling, 1:500), c-Myc (Santa Cruz Biotechnology, 1:500) Col I (Abcam, 1:500), Col II (Santa Cruz Biotechnology, 1:100), Col IV (Abcam, 1:500), E-cad (Santa Cruz Biotechnology, 1:500), FN (Abcam, 1:1000), HOPX (Santa Cruz Biotechnology, 1:200), Isolectin B4, FITC-conjugate/IsoB4 (Sigma, 1:500), Laminin (Sigma, 1:1000), LAMP3 (1:100, Imgenex), MMP2 (Millipore, 1:500), MYC (Santa Cruz Biotechnology (9E10), 1:1000), MYH10 (Sigma, 1:500), NG2 (Millipore, 1:500), NKX2-1 (Santa Cruz Biotechnology, 1:200), PCNA (DAKO, 1:200), PDGFR- $\beta$ (R\&D Systems, 1:200), PDPN/T1 $\alpha$ (Developmental Studies Hybridoma Bank, 1:20), PECAM/CD31 (Developmental Studies Hybridoma Bank, 1:1), phalloidin, rhodamine-conjugate (ThermoFisher, 1:1000), phospho-histone H3 (Cell Signaling, 1:1000), RAGE (R\&D Systems, 1:200), SCGB1A1/CC10 (Millipore, 1:1000), SFTPC/ProSP-C (Millipore, 1:1000), a-SMA (Sigma, 1:1000), THBS1 (Santa Cruz Biotechnology, 1:200), THBS2 (Santa Cruz Biotechnology, 1:200), tropoelastin (Abcam, 1:1000), and VE-cadherin (BD Biosciences, 1:500).

RT-qPCR and western blot analysis. Total RNA and cDNA were obtained from fetal and postnatal lungs using TRIzol reagent (Invitrogen) and Superscript III reverse transcriptase (Invitrogen), respectively, according to the manufacturer's instructions. For quantitative reverse transcription PCR, the CFX Connect RealTime system (Bio-Rad) and DyNAmo colorFlash SYBR green qPCR kit (ThermoFisher Scientific) were used. The primer sequences used for qPCR are shown in 
Supplementary Table 2. The Ct values are shown in Supplementary Table 3. For western blots, protein extracts were prepared from fetal and postnatal lungs by using NP-40 buffer (150 mM NaCl, $1.0 \% \mathrm{NP}-40,50 \mathrm{mM}$ Tris $\mathrm{pH} 8.0,1 \mathrm{mM}$ phenylmethane sulfonyl fluoride). Western blotting was performed according to standard protocols. Primary antibodies were incubated overnight at $4{ }^{\circ} \mathrm{C}$ in $3 \%$ skim milk. The following antibodies were used: $\alpha$-actin (Sigma, 1:2000), Col I (1:2000), GAPDH (Cell Signaling, 1:2000), FN (1:2000), MMP2 (Millipore, 1:2000), MMP9 (Millipore, 1:2000), MT1-MMP/MMP14 (Santa Cruz Biotechnology, 1:500), MYH10 (1:2000), THBS2 (1:500), and tropoelastin (1:2000). All uncropped images related to western blotting data are available in Supplementary Fig. 10.

Histology and head skeletal staining. Whole lungs were fixed in $4 \%$ formaldehyde, embedded in paraffin, and sectioned at a thickness of $5 \mu \mathrm{m}$. For cryosections, fixed tissues were embedded in optimal cutting temperature (OCT) compound and $10 \mu \mathrm{m}$ thick coronal sections were collected. Hematoxylin and eosin (H\&E) and PAS staining were performed according to standard protocols. For head skeletal staining, newborn mice were deskinned, dehydrated in $95 \%$ ethanol for 1 day and acetone for 1 day. Skeletons were stained with $0.03 \%$ alcian blue (Sigma) and $0.005 \%$ Alizarin red (Sigma). After staining, samples were cleared in $2 \%$ potassium hydroxide and transferred to $50 \%$ glycerol for 2 days.

TEM. For TEM analysis, tissues were fixed in $3 \%$ glutaraldehyde, followed by $4 \%$ osmium tetraoxide $\left(\mathrm{OsO}_{4}\right)$ in $0.1 \mathrm{mM}$ sodium cacodylate buffer ( $\mathrm{pH}$ 7.4). After dehydration in ethanol and propylene oxide, the samples were embedded in Epon 812. Ultrathin sections were cut with a diamond knife and stained with uranyl acetate and lead citrate, and examined with a JEM1400 electron microscope (JEOL).

Cell culture, chemical treatments, and transfections. NIH3T3 cells and Myh10 null mouse embryonic fibroblasts were provided by Dr. Stefan Offermanns and Dr. Robert S. Adelstein, respectively. Embryonic fibroblasts, and NIH3T3 cells were maintained at $37^{\circ} \mathrm{C}$ in a $5 \% \mathrm{CO}_{2}$ incubator in Dulbecco's modified Eagle's medium (DMEM), supplemented with $10 \%$ fetal bovine serum, $50 \mathrm{~g} / \mathrm{ml}$ streptomycin, $50 \mathrm{U} /$ $\mathrm{ml}$ penicillin, and $250 \mathrm{ng} / \mathrm{ml}$ amphotericin B. Cells were treated with DMSO, Blebbistatin $(10 \mu \mathrm{M})$, GM6001 $(10 \mu \mathrm{M})$, and MMP2 inhibitor I (Santa Cruz Biotechnology, SC-204092, $10 \mu \mathrm{M}$ ) at $37^{\circ} \mathrm{C}$ in a $5 \% \mathrm{CO}_{2}$ incubator for $24-48 \mathrm{~h}$. Transfection was performed using Lipofectamine 2000 transfection reagent (ThermoFisher Scientific) according to manufacturer's instructions.

MMP activity assays. For gelatin zymography, lung tissues were homogenized in homogenization buffer (50 mM Tris-HCl, $0.5 \%$ Triton X-100, pH 7.4) and supernatant containing gelatinases was collected following centrifugation. For the MMP activity assay, a standard commercial assay kit (Abcam) was used to detect the general activity of MMPs in Myh10 null and wild-type mouse embryonic fibroblasts according to manufacturer's instructions. Cells were cultured at $37^{\circ} \mathrm{C}$ in a $5 \% \mathrm{CO}_{2}$ incubator in serum-free DMEM for $48 \mathrm{~h}$. Cell lysates and conditioned media were incubated with a fluorescent substrate and fluorescence was measured using a fluorescence microplate reader (FLUOstar Omega, BMG LABTECH).

siRNA knockdowns. siRNAs for EGFP (EHUEGFP, Sigma) and Mmp2 (EMU04861, Sigma) were transfected using RNAiMAX transfection reagent (13778, Life Technologies) according to the manufacturer's instructions.

FN assembly assay. Myh10 null and wild-type mouse embryonic fibroblasts were cultured for $24 \mathrm{~h}$ in complete growth medium. Cells were then kept for $24 \mathrm{~h}$ in starvation medium and scraped into $2 \%$ Deoxycholate (DOC) lysis buffer. DOCsoluble material was removed, and sodium dodecyl sulfate-polyacrylamide gel electrophoresis (SDS-PAGE) sample buffer was added to these lysates. DOCinsoluble material was resuspended in solubilization buffer and treated with SDSPAGE sample buffer. All samples were boiled for $5 \mathrm{~min}$ before western blot analysis.

Transcriptomic analysis (RNAseq). For RNAseq, total RNA was isolated from E17 lungs (left lobes) of two $M y h 10^{+/+}$and two Myh10-/- mice using Trizol (Life Technologies) combined with on-column DNase digestion (TURBO DNAse, Ambion) to avoid contamination by genomic DNA. RNA and library preparation integrity were verified with a BioAnalyzer 2100 (Agilent) or LabChip Gx Touch 24 (Perkin Elmer). In all, $3 \mu \mathrm{g}$ of total RNA was used as input for Truseq Stranded mRNA Library preparation following the low sample protocol (Illumina). Sequencing was performed on a NextSeq500 instrument (Illumina) using v2 chemistry, resulting in $30 \mathrm{M}-50 \mathrm{M}$ reads per library, with $1 \times 75$ bp single-end setup. The resulting raw reads were assessed for quality, adapter content, and duplication rates with FastQC (http://www.bioinformatics.babraham.ac.uk/ projects/fastqc). Reaper version 15-065 was employed to trim reads after a quality drop below a mean of Q20 in a window of 20 nucleotides. Only reads between 15 and 75 nucleotides were considered for further analyses. Trimmed and filtered reads were aligned to the Ensembl mouse genome version mm10 (GRCm38) using STAR 2.4.0a with the parameter "--outFilterMismatchNoverLmax 0.1" to increase the maximum ratio of mismatches to mapped length to $10 \%$. The number of reads aligning to genes was counted with the featureCounts 1.5.1 tool from the Subread package. Only reads mapping at least partially inside exons were admitted and aggregated per gene. Reads overlapping multiple genes or aligning to multiple regions were excluded. Differentially expressed genes (DEGs) were identified using DESeq2 version 1.14.1. Only genes with a minimum fold change of $\pm 2(\log 2 \pm 1)$, a maximum Benjamini-Hochberg corrected $P$-value of 0.05 , and a minimum combined mean of five reads were considered to be significantly differentially expressed. The Ensembl annotation was enriched with UniProt data based on Ensembl gene identifiers (Activities at the Universal Protein Resource (UniProt)). To examine the biological significance of the DEGs, we carried out a Gene Ontology (GO) enrichment analysis using the online tool Database for Annotation, Visualization and Integrated Discovery (DAVID) Bioinformatics Resource v6.8 (https:// david.ncifcrf.gov/home.jsp) ${ }^{66}$ (Supplementary Data 1).

Proteomic analysis (mass spectrometry). Triplicate analyses were performed as follows: Sample preparation. Cells were lysed by heating to $70^{\circ} \mathrm{C}$ for $10 \mathrm{~min}$ in SDS buffer (4\% SDS in $0.1 \mathrm{M} \mathrm{Tris/HCl,} \mathrm{pH} \mathrm{7.6).} \mathrm{DNA} \mathrm{was} \mathrm{sheared} \mathrm{by} \mathrm{sonication} \mathrm{and}$ cell debris was removed by centrifugation at $16,000 \mathrm{~g}$ for $10 \mathrm{~min}$. A colorimetric $660 \mathrm{~nm}$ protein assay (Pierce) was used to determine the concentration of solubilized proteins in the resulting supernatants. Proteins were subsequently precipitated by addition of four volumes of acetone and incubation at $-20^{\circ} \mathrm{C}$ overnight, followed by pelleting at $14,000 \mathrm{~g}$ for $10 \mathrm{~min}$ and washing the pellet with $90 \%$ acetone. Samples were dried to remove acetone completely and dissolved in urea buffer ( $6 \mathrm{M}$ urea, $2 \mathrm{M}$ thiourea, $10 \mathrm{mM}$ HEPES, $\mathrm{pH}$ 8.0). Enzymatic digestion of proteins was performed by in-solution digestion. Briefly, protein disulfide bonds were reduced with $4 \mathrm{mM}$ dithiothreitol and alkylated with $20 \mathrm{mM}$ iodoacetamide. Proteins were then cleaved using Lys-C (50:1 protein-to-enzyme ratio) (Wako Chemicals $\mathrm{GmbH}$ ) at room temperature for $3 \mathrm{~h}$, followed by overnight trypsination (50:1 protein-to-enzyme ratio) (Serva) at room temperature. Peptide labeling by reductive dimethylation was performed as previously described ${ }^{67}$. Peptide concentration in trypsin-digested samples was measured using the Fluorimetric Peptide Assay (Pierce). Samples containing equal amounts of peptides $(75 \mu \mathrm{g})$ were subjected to the dimethyl (in-solution) labeling protocol. In brief, peptide $\mathrm{N}$ termini and lysine residues were methylated for $1 \mathrm{~h}$ at RT by formaldehyde- $\mathrm{H}_{2}$ and cyanoborohydride (light, control mouse fibroblasts) and formaldehyde $-{ }^{3} \mathrm{C}-\mathrm{D}_{2}$ and cyanoborodeuteride (heavy, Myh10-KD), respectively (all reagents: Sigma). The reaction was quenched by acidification and differentially labeled samples were mixed in 1:1 ratio. Mixed samples were fractionated using the high $\mathrm{pH}$ reversedphase peptide fractionation kit (Pierce) according to manufacturer's instructions.

Liquid chromatography/tandem mass spectrometry. For mass spectrometry analysis, fractionated peptides were reconstituted in $10 \mu \mathrm{l}$ of solvent $\mathrm{A}(0.1 \%$ formic acid). Peptides were separated using an UHPLC system (EASY-nLC 1000, ThermoFisher Scientific) and $20 \mathrm{~cm}$ in-house packed C18 silica columns $(1.9 \mu \mathrm{m}$ C18 beads, Dr. Maisch GmbH) coupled in line to a QExactive HF orbitrap mass spectrometer (ThermoFisher Scientific) using an electrospray ionization source. The gradient employed used linearly increasing concentrations of solvent B $(90 \%$ acetonitrile, $1 \%$ formic acid) over solvent A ( $5 \%$ acetonitrile, $1 \%$ formic acid) from 5 to $30 \%$ over $215 \mathrm{~min}$, from 30 to $60 \%$, from 60 to $95 \%$ and from 95 to $5 \%$ for 5 min each, followed by re-equilibration with $5 \%$ of solvent $B$. The flow rate was set to a constant $400 \mathrm{nl} / \mathrm{min}$. Full MS spectra were acquired for a mass range of $300-1750 \mathrm{~m} / \mathrm{z}$ with a resolution of 60,000 at $200 \mathrm{~m} / \mathrm{z}$. The ion injection target was set to $3 \times 10^{6}$ and the maximum injection time limited to $20 \mathrm{~ms}$. Ions were fragmented by higher energy collision dissociation using a normalized collision energy of 27, an isolation window width of $2.2 \mathrm{~m} / \mathrm{z}$ and an ion injection target of $5 \times 10^{5}$ with a maximum injection time of $20 \mathrm{~ms}$. Precursors with unassigned charge state and a charge state of 1 were excluded from selection for fragmentation. The duration of dynamic exclusion was set to $20 \mathrm{~s}$. Resulting tandem mass spectra (MS/MS) were acquired with a resolution of 15,000 at $200 \mathrm{~m} / \mathrm{z}$ using data dependent mode with a loop count of 15 (top15).

Data analysis. MS raw data were processed by MaxQuant (1.5.6.5) using the Uniprot mouse database (as of 20 April 2017) containing 89,316 entries and the following parameters: a maximum of two missed cleavages, mass tolerance of 4.5 ppm for the main search, trypsin as the digesting enzyme, carbamidomethylation of cysteins as a fixed modification, and oxidation of methionine, as well as acetylation of the protein $\mathrm{N}$-terminus as variable modifications. For protein quantification based on dimethyl labeling, isotope labels were configured for peptide $\mathrm{N}$-termini and lysine residues with a monoisotopic mass increase of 28.0313 and $36.0757 \mathrm{Da}$ for the light and heavy labels, respectively. Peptides with a minimum of seven amino acids and at least one unique peptide were included in protein identification. MaxQuant was instructed to filter for $1 \%$ false discovery rate on both the peptide and protein levels. Only proteins with at least two peptides and one unique peptide were considered as identified and were used for further data analysis. The resulting data contained 2949 protein groups and was subjected to differential expression analysis using the in-house R package autonomics (https:// bitbucket.org/graumannlabtools/autonomics; version 1.0.21), which makes heavy use of functionality provided by the limma package ${ }^{68}$. The analysis considered protein groups that were quantified in at least one of the triplicate samples, with lack of completeness strongly penalized by the underlying Bayesian-moderated $t$ testing. To examine the biological significance of the differentially expressed 
proteins, we used autonomics to carry out overrepresentation analysis for GO terms using a Fisher's exact test (Supplementary Data 2).

Human emphysematous lung samples. Emphysematous lungs and healthy control lungs were provided by the UGMLC Giessen Biobank, which is a member of the DZL Platform Biobanking. All patients were diagnosed with COPD/ emphysema by pulmonary function testing, computed tomography scans, and pathological examination from resected lung specimens. The severity of COPD was classified according to the Global Initiative on Obstructive Lung Disease (GOLD) staging system and all subjects belonged to GOLD IV. The severity of emphysema was assessed by pathological inspection of histological samples and graded according to the method as previously described ${ }^{69}$. The study protocol was approved by the Ethics Committee of the Justus-Liebig-University School of Medicine (no. 58/2015), and informed consent was obtained in written form from each patient. All human studies were performed in adherence to the relevant ethical guidelines.

Morphometric analyses. Pictures of H\&E-stained distal lungs were acquired using a Zeiss widefield microscope (Axiolmager). Alveolar spaces, thickness of alveolar walls, and length of secondary septae were measured using Image (https://imagej. nih.gov/ij/) according to the American Thoracic Society guidelines ${ }^{70}$. A minimum of five representative, non-overlapping fields of view from lungs of at least three mice from each group were evaluated.

Statistical analysis. Data are presented as mean \pm s.e.m of at least three biological replicates. Two-tailed Student's $t$-tests were used to assess significance. A $P$-value of $<0.05$ was considered significant.

\section{Data availability}

RNA-sequencing data from this study have been deposited in GEO under accession code GSE1 19399 (Supplementary Data 1). Proteomic analysis data from this study have been deposited to the ProteomeXchange Consortium via the PRIDE partner repository with the dataset identifier PXD011139 (Supplementary Data 2). All remaining data will be available from the corresponding author upon reasonable request.

Received: 27 December 2017 Accepted: 25 September 2018 Published online: 02 November 2018

\section{References}

1. Burri, P. H. Fetal and postnatal development of the lung. Annu. Rev. Physiol. 46, 617-628 (1984)

2. Cardoso, W. V. \& Lu, J. Regulation of early lung morphogenesis: questions, facts and controversies. Development 133, 1611-1624 (2006).

3. Warburton, D. et al. Lung organogenesis. Curr. Top. Dev. Biol. 90, 73-158 (2010).

4. Rock, J. R. \& Hogan, B. L. Epithelial progenitor cells in lung development, maintenance, repair, and disease. Annu. Rev. Cell. Dev. Biol. 27, 493-512 (2011).

5. Herriges, M. \& Morrisey, E. E. Lung development: orchestrating the generation and regeneration of a complex organ. Development 141, 502-513 (2014).

6. Branchfield, K. et al. A three-dimensional study of alveologenesis in mouse lung. Dev. Biol. 409, 429-441 (2016).

7. McCulley, D., Wienhold, M. \& Sun, X. The pulmonary mesenchyme directs lung development. Curr. Opin. Genet. Dev. 32, 98-105 (2015).

8. Vicente-Manzanares, M., Ma, X., Adelstein, R. S. \& Horwitz, A. R. Nonmuscle myosin II takes centre stage in cell adhesion and migration. Nat. Rev. Mol. Cell Biol. 10, 778-790 (2009).

9. Heissler, S. M. \& Manstein, D. J. Nonmuscle myosin-2: mix and match. Cell. Mol. Life Sci. 70, 1-21 (2013).

10. Newell-Litwa, K. A., Horwitz, R. \& Lamers, M. L. Non-muscle myosin II in disease: mechanisms and therapeutic opportunities. Dis. Model Mech. 8, 1495-1515 (2015).

11. Berg, J. S., Powell, B. C. \& Cheney, R. E. A millennial myosin census. Mol. Biol. Cell. 12, 780-794 (2001).

12. Conti, M. A. \& Adelstein, R. S. Nonmuscle myosin II moves in new directions. J. Cell. Sci. 121, 11-18 (2008).

13. Ma, X. et al. Ablation of nonmuscle myosin II-B and II-C reveals a role for nonmuscle myosin II in cardiac myocyte karyokinesis. Mol. Biol. Cell. 21, 3952-3962 (2010).

14. Plosa, E. J., Gooding, K. A., Zent, R. \& Prince, L. S. Nonmuscle myosin II regulation of lung epithelial morphology. Dev. Dyn. 241, 1770-1781 (2012).
15. Decramer, M., Janssens, W. \& Miravitlles, M. Chronic obstructive pulmonary disease. Lancet 379, 1341-1351 (2012).

16. Rabe, K. F. \& Watz, H. Chronic obstructive pulmonary disease. Lancet 389, 1931-1940 (2017)

17. Fischer, A. \& du Bois, R. Interstitial lung disease in connective tissue disorders Lancet 380, 1148-1148 (2012).

18. Taraseviciene-Stewart, L. \& Voelkel, N. F. Molecular pathogenesis of emphysema. J. Clin. Invest. 118, 394-402 (2008).

19. Marcelino, M. Y. et al. Animal models in chronic obstructive pulmonary disease-an overview. Exp. Lung Res. 40, 259-271 (2014).

20. Perez-Rial, S., Giron-Martinez, A. \& Peces-Barba, G. Animal models of chronic obstructive pulmonary disease. Arch. Bronconeumol. 51, 121-127 (2015).

21. Justice, M. J., Noveroske, J. K., Weber, J. S., Zheng, B. \& Bradley, A. Mouse ENU mutagenesis. Hum. Mol. Genet. 8, 1955-1963 (1999).

22. Tullio, A. N. et al. Nonmuscle myosin II-B is required for normal development of the mouse heart. Proc. Natl. Acad. Sci. USA 94, 12407-12412 (1997).

23. Barron, L., Gharib, S. A. \& Duffield, J. S. Lung pericytes and resident fibroblasts: busy multitaskers. Am. J. Pathol. 186, 2519-2531 (2016).

24. Treutlein, B. et al. Reconstructing lineage hierarchies of the distal lung epithelium using single-cell RNA-seq. Nature 509, 371-375 (2014).

25. Laresgoiti, U. et al. Lung epithelial tip progenitors integrate glucocorticoidand STAT3-mediated signals to control progeny fate. Development 143, 3686-3699 (2016)

26. Ridsdale, R. \& Post, M. Surfactant lipid synthesis and lamellar body formation in glycogen-laden type II cells. Am. J. Physiol. Lung Cell. Mol. Physiol. 287, L743-L751 (2004)

27. Takeda, K., Kishi, H., Ma, X., Yu, Z. X. \& Adelstein, R. S. Ablation and mutation of nonmuscle myosin heavy chain II-B results in a defect in cardiac myocyte cytokinesis. Circ. Res. 93, 330-337 (2003).

28. Ma, X., Bao, J. \& Adelstein, R. S. Loss of cell adhesion causes hydrocephalus in nonmuscle myosin II-B-ablated and mutated mice. Mol. Biol. Cell. 18, 2305-2312 (2007)

29. Shutova, M., Yang, C., Vasiliev, J. M. \& Svitkina, T. Functions of nonmuscle myosin II in assembly of the cellular contractile system. PLoS ONE. 7, e40814 (2012).

30. Zhang, W. et al. Spatial-temporal targeting of lung-specific mesenchyme by a Tbx4 enhancer. BMC. Biol. 11, 111 (2013).

31. Bonnans, C., Chou, J. \& Werb, Z. Remodelling the extracellular matrix in development and disease. Nat. Rev. Mol. Cell Biol. 15, 786-801 (2014)

32. Rao, Y., Hao, R., Wang, B. \& Yao, T. P. A Mec17-myosin II effector axis coordinates microtubule acetylation and actin dynamics to control primary cilium biogenesis. PLoS ONE. 9, el14087 (2014)

33. Hong, H., Kim, J. \& Kim, J. Myosin heavy chain 10 (MYH10) is required for centriole migration during the biogenesis of primary cilia. Biochem. Biophys. Res. Commun. 461, 180-185 (2015).

34. Cardoso, W. V. Molecular regulation of lung development. Annu. Rev. Physiol 63, 471-494 (2001)

35. Lu P. F., Takai K., Weaver V. M. \& Werb Z. Extracellular matrix degradation and remodeling in development and disease. Cold Spring Harb. Perspect. Biol. 3, a005058 (2011).

36. Page-McCaw, A., Ewald, A. J. \& Werb, Z. Matrix metalloproteinases and the regulation of tissue remodelling. Nat. Rev. Mol. Cell Biol. 8, 221-233 (2007).

37. Rodriguez, D., Morrison, C. J. \& Overall, C. M. Matrix metalloproteinases: what do they not do? New substrates and biological roles identified by murine models and proteomics. Bba-Mol. Cell Res. 1803, 39-54 (2010).

38. Greenlee, K. J., Werb, Z. \& Kheradmand, F. Matrix metalloproteinases in lung: multiple, multifarious, and multifaceted. Physiol. Rev. 87, 69-98 (2007).

39. Lo, C. M. et al. Nonmuscle myosin IIb is involved in the guidance of fibroblast migration. Mol. Biol. Cell. 15, 982-989 (2004).

40. Kalson, N. S. et al. Nonmuscle myosin II powered transport of newly formed collagen fibrils at the plasma membrane. Proc. Natl. Acad. Sci. USA 110, E4743-E4752 (2013).

41. Wang, Z. et al. Interleukin-lbeta induces migration of rat arterial smooth muscle cells through a mechanism involving increased matrix metalloproteinase-2 activity. J. Surg. Res. 169, 328-336 (2011)

42. Kielty, C. M., Sherratt, M. J. \& Shuttleworth, C. A. Elastic fibres. J. Cell. Sci. 115, 2817-2828 (2002).

43. Yanagisawa, H. \& Davis, E. C. Unraveling the mechanism of elastic fiber assembly: the roles of short fibulins. Int. J. Biochem. Cell. Biol. 42, 1084-1093 (2010).

44. Adams, J. C. \& Lawler, J. The thrombospondins. Cold Spring Harb. Perspect. Biol. 3, a009712 (2011)

45. Calabro, N. E., Kristofik, N. J. \& Kyriakides, T. R. Thrombospondin-2 and extracellular matrix assembly. Bba-Gen. Subj. 1840, 2396-2402 (2014).

46. Resovi, A., Pinessi, D., Chiorino, G. \& Taraboletti, G. Current understanding of the thrombospondin-1 interactome. Matrix Biol. 37, 83-91 (2014). 
47. Ma, X. et al. Conditional ablation of nonmuscle myosin II-B delineates heart defects in adult mice. Circ. Res. 105, 1102-1109 (2009).

48. Kramann, R. et al. Perivascular Gli1+progenitors are key contributors to injury-induced organ fibrosis. Cell. Stem. Cell. 16, 51-66 (2015).

49. Hellewell A. L., Gong X. Y., Scharich K., Christofidou E. D. \& Adams J. C. Modulation of the extracellular matrix patterning of thrombospondins by actin dynamics and thrombospondin oligomer state. Biosci. Rep. 35, e00218 (2015).

50. Riessen, R., Kearney, M., Lawler, J. \& Isner, J. M. Immunolocalisation of thrombospondin-1 in human atherosclerotic and restenotic arteries. Am. Heart J. 135, 357-364 (1998).

51. Zaidel-Bar, R., Guo, Z. H. \& Luxenburg, C. The contractome - a systems view of actomyosin contractility in non-muscle cells. J. Cell. Sci. 128, 2209-2217 (2015).

52. Pandya, P., Orgaz, J. L. \& Sanz-Moreno, V. Actomyosin contractility and collective migration: may the force be with you. Curr. Opin. Cell Biol. 48, 87-96 (2017).

53. Finlay, G. A., O’Donnell, M. D., O’Connor, C. M., Hayes, J. P. \& FitzGerald, M. X. Elastin and collagen remodeling in emphysema. A scanning electron microscopy study. Am. J. Pathol. 149, 1405-1415 (1996).

54. Birukov, K. G. Balancing between stiff and soft: a life-saving compromise for lung epithelium in lung injury. J. Appl. Physiol. (1985) 117, 1213-1214 (2014).

55. Takahashi, A., Majumdar, A., Parameswaran, H., Bartolak-Suki, E. \& Suki, B. Proteoglycans maintain lung stability in an elastase-treated mouse model of emphysema. Am. J. Respir. Cell Mol. Biol. 51, 26-33 (2014).

56. Kumper, S. \& Marshall, C. J. ROCK-driven actomyosin contractility induces tissue stiffness and tumor growth. Cancer Cell. 19, 695-697 (2011).

57. Humphrey, J. D., Dufresne, E. R. \& Schwartz, M. A. Mechanotransduction and extracellular matrix homeostasis. Nat. Rev. Mol. Cell Biol. 15, 802-812 (2014).

58. Castaldi, P. J. et al. Genome-wide association identifies regulatory loci associated with distinct local histogram emphysema patterns. Am. J. Respir. Crit. Care. Med. 190, 399-409 (2014).

59. Golpon, H. A. et al. Emphysema lung tissue gene expression profiling. Am. J. Respir. Cell Mol. Biol. 31, 595-600 (2004).

60. Elkington, P. T. \& Friedland, J. S. Matrix metalloproteinases in destructive pulmonary pathology. Thorax 61, 259-266 (2006).

61. Baraldo, S. et al. Matrix metalloproteinase- 2 protein in lung periphery is related to COPD progression. Chest 132, 1733-1740 (2007).

62. Wright, J. L., Cosio, M. \& Churg, A. Animal models of chronic obstructive pulmonary disease. Am. J. Physiol. Lung Cell. Mol. Physiol. 295, L1-L15 (2008).

63. Navratilova, Z., Kolek, V. \& Petrek, M. Matrix metalloproteinases and their inhibitors in chronic obstructive pulmonary disease. Arch. Immunol. Ther. Exp. (Warsz.) 64, 177-193 (2016).

64. Salinger A. P. \& Justice M. J. Mouse mutagenesis using N-ethyl-N-nitrosourea (ENU). Cold Spring Harb. Protoc. doi:10.1101/pdb.prot4985 (2008).

65. Yin, W. et al. The potassium channel KCNJ13 is essential for smooth muscle cytoskeletal organization during mouse tracheal tubulogenesis. Nat. Commun. 9, 2815 (2018).

66. Huang, D. W., Sherman, B. T. \& Lempicki, R. A. Systematic and integrative analysis of large gene lists using DAVID bioinformatics resources. Nat. Protoc. 4, 44-57 (2009).

67. Boersema, P. J., Raijmakers, R., Lemeer, S., Mohammed, S. \& Heck, A. J. Multiplex peptide stable isotope dimethyl labeling for quantitative proteomics. Nat. Protoc. 4, 484-494 (2009).

68. Ritchie, M. E. et al. limma powers differential expression analyses for RNAsequencing and microarray studies. Nucleic Acids Res. 43, e47 (2015).
69. Thurlbeck, W. M. et al. A comparison of three methods of measuring emphysema. Hum. Pathol. 1, 215-226 (1970).

70. Hsia, C. C., Hyde, D. M., Ochs, M. \& Weibel, E. R., ATS/ERS Joint Task Force on Quantitative Assessment of Lung Structure. An official research policy statement of the American Thoracic Society/European Respiratory Society: standards for quantitative assessment of lung structure. Am. J. Respir. Crit. Care. Med. 181, 394-418 (2010).

\section{Acknowledgements}

We thank Saverio Bellusci for the Shh-Cre and Gli1-CreER ${ }^{T 2}$ mice, Nina Wettschureck for the Tek-CreER ${ }^{T 2}$ mice, and Birgit Spiznagel for the CMV-Cre mice. We thank Konstantinos Gkatzis, Arica Beisaw, and Viola Graef for critically reading the manuscript. This work was supported by funds from the Max Planck Society to D.Y.R.S.

\section{Author contributions}

H.-T.K. designed and performed experiments, and wrote the manuscript; W.Y., P.P., F.G., B.G., and C.B. contributed to data analysis and experiments; H.-T.K., Y.-J.J., and S.O. designed and performed in vitro studies; S.K. performed TEM analysis; A.M.S., A.M.B., J.G., and H.-T.K. performed mass spectrometry and data analysis; S.G. and H.-T.K. performed RNA-sequencing and data analysis; J.P. and M.L. performed data analysis for whole-exome sequencing; X.M. and R.S.A. provided Myh10 flox mice and Myh10 KO embryonic fibroblasts; C.R. and A.G. provided human patient samples; D.Y. R.S. conceived the ENU screen, enabled and supervised the project, analyzed data, and edited the manuscript. All authors commented on the manuscript.

\section{Additional information}

Supplementary Information accompanies this paper at https://doi.org/10.1038/s41467018-06833-7.

Competing interests: The authors declare no competing interests.

Reprints and permission information is available online at http://npg.nature.com/ reprintsandpermissions/

Publisher's note: Springer Nature remains neutral with regard to jurisdictional claims in published maps and institutional affiliations.

pen Access This article is licensed under a Creative Commons Attribution 4.0 International License, which permits use, sharing, adaptation, distribution and reproduction in any medium or format, as long as you give appropriate credit to the original author(s) and the source, provide a link to the Creative Commons license, and indicate if changes were made. The images or other third party material in this article are included in the article's Creative Commons license, unless indicated otherwise in a credit line to the material. If material is not included in the article's Creative Commons license and your intended use is not permitted by statutory regulation or exceeds the permitted use, you will need to obtain permission directly from the copyright holder. To view a copy of this license, visit http://creativecommons.org/ licenses/by/4.0/.

(C) The Author(s) 2018 